\title{
Consistent description of ion-specificity in bulk and at interfaces by solvent implicit simulations and mean-field theory
}

Cite as: J. Chem. Phys. 153, 034103 (2020); https://doi.org/10.1063/5.0016103

Submitted: 02 June 2020 . Accepted: 22 June 2020 . Published Online: 15 July 2020

(iD) Alexandre P. dos Santos, (D) Yuki Uematsu, Alexander Rathert, (i) Philip Loche, and (iD) Roland R. Netz
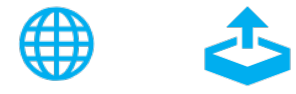

\section{ARTICLES YOU MAY BE INTERESTED IN}

Simulations of activities, solubilities, transport properties, and nucleation rates for aqueous electrolyte solutions

The Journal of Chemical Physics 153, 010903 (2020); https://doi.org/10.1063/5.0012102

Simulations of electrolyte between charged metal surfaces

The Journal of Chemical Physics 153, 044121 (2020); https://doi.org/10.1063/5.0012073

Molecular modeling of aqueous electrolytes at interfaces: Effects of long-range dispersion forces and of ionic charge rescaling

The Journal of Chemical Physics 152, 241102 (2020); https://doi.org/10.1063/5.0011058

Challenge us.

What are your needs for periodic signal detection?

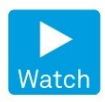

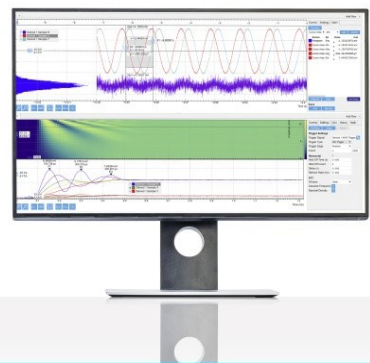

- Zurich

Instruments 


\title{
Consistent description of ion-specificity in bulk and at interfaces by solvent implicit simulations and mean-field theory
}

\author{
Cite as: J. Chem. Phys. 153, 034103 (2020); doi: 10.1063/5.0016103 \\ Submitted: 2 June 2020 - Accepted: 22 June 2020 • \\ Published Online: 15 July 2020
}

\begin{abstract}
Alexandre P. dos Santos, ${ }^{1,2, a)}$ (D) Yuki Uematsu, ${ }^{3, b)}$ (D) Alexander Rathert, ${ }^{2, b)}$ Philip Loche, ${ }^{2, b)}$ (D) and Roland R. Netz ${ }^{2, b)}$ (D)

\section{AFFILIATIONS}

${ }^{1}$ Instituto de Física, Universidade Federal do Rio Grande do Sul, Caixa Postal 15051, 91501-970 Porto Alegre, RS, Brazil

${ }^{2}$ Fachbereich Physik, Freie Universität Berlin, 14195 Berlin, Germany

${ }^{3}$ Department of Physics, Kyushu University, 819-0395 Fukuoka, Japan
\end{abstract}

\begin{abstract}
a) Author to whom correspondence should be addressed: alexandre.pereira@ufrgs.br
${ }^{b}$ Electronic addresses: uematsu.yuki@phys.kyushu-u.ac.jp; a.rathert@fu-berlin.de; ploche@physik.fu-berlin.de; and rnetz@physik.fu-berlin.de
\end{abstract}

\begin{abstract}
Solvent-implicit Monte Carlo (MC) simulations and mean-field theory are used to predict activity coefficients and excess interfacial tensions for $\mathrm{NaF}, \mathrm{NaCl}, \mathrm{NaI}, \mathrm{KF}, \mathrm{KCl}$, and $\mathrm{KI}$ solutions in good agreement with experimental data over the entire experimentally available concentration range. The effective ionic diameters of the solvent-implicit simulation model are obtained by fits to experimental activity coefficient data. The experimental activity coefficients at high salt concentrations are only reproduced if the ion-specific concentration-dependent decrement of the dielectric constant is included. The dielectric-constant dependent contribution of the single-ion solvation free energy to the activity coefficient is significant and is included. To account for the ion-specific excess interfacial tension of salt solutions, in addition to nonideal solution effects and the salt-concentration-dependent dielectric decrement, an ion-specific ion-interface interaction must be included. This ion-interface interaction, which acts in addition to the dielectric image-charge repulsion, is modeled as a box potential, is considerably more long-ranged than the ion radius, and is repulsive for all ions considered except iodide, in agreement with previous findings and arguments. By comparing different models that include or exclude bulk non-ideal solution behavior, dielectric decrement effects, and ioninterface interaction potentials, we demonstrate how bulk and interfacial ion-specific effects couple and partially compensate each other. Our MC simulations, which correctly include ionic correlations and interfacial dielectric image-charge repulsion, are used to determine effective ion-surface interaction potentials that can be used in a modified Poisson-Boltzmann theory.
\end{abstract}

Published under license by AIP Publishing. https://doi.org/10.1063/5.0016103

\section{INTRODUCTION}

The ionic specificity observed in interfacial tension data, ${ }^{1-7}$ critical coagulation or micellization concentration data of colloidal and micellar suspensions, ${ }^{8-16}$ surface interactions, ${ }^{17,18}$ and protein structure and kinetics ${ }^{19,20}$ still poses a number of questions. ${ }^{21-24}$ This is so because in all ion-specific phenomena that involve surfaces in contact with salt solutions, three distinct effects are intimately coupled: (i) Ions interact with interfaces not only by universal Coulomb and image-charge forces but also by ion-specific force contributions that involve hydration effects and van der Waals interactions. (ii) The bulk ion chemical potential depends on salt concentration with pronounced variations among different ions, and this is at the heart of bulk ion specificity and sensitively influences the ionic surface adsorption equilibrium. (iii) Finally, the dielectric constant of salt solutions decreases with rising salt concentration in a manner that is again different for different ions. The dielectric constant not only influences the bulk ion chemical potential but also the ion-interface dielectric image-charge interactions. All three abovementioned effects have been amply treated separately in the literature, but an investigation of how these effects conspire to produce ion-specific effects at interfaces has been largely missing. 
In early modeling approaches, the dispersion or van der Waals interaction between ions and the air-water interface was suggested to be responsible for ion-specific effects seen in air-water interfacial tension data. ${ }^{25}$ Ion-specific interactions between ions and the air-water interface that reproduced and explained the experimental trends were in water-explicit simulations first observed with polarizable force fields, ${ }^{26,27}$ and such simulations also demonstrated the enhanced interface activity of iodide. ${ }^{28-31}$ The contribution of ionic polarizability to ion-interface interactions was investigated in a number of subsequent theoretical and simulation works. As a general trend, it was seen that small ions with tightly bound hydration water are strongly repelled from the electrolyte-air interface, while larger ions with loosely bound hydration water are less strongly repelled or can even adsorb at the interface. ${ }^{32-35}$

It was later shown that well-parametrized non-polarizable force-field simulations can also account for ion-specific ioninterface interactions and, in conjunction with mean-field Poisson-Boltzmann modeling, reproduce experimental excess surface tensions. ${ }^{36,37}$ More recently, the salt-concentration-dependent air-water interfacial tension was directly extracted from waterexplicit molecular dynamics simulations and shown to reproduce the experimental difference between acids, which lower the interfacial tension, and bases, which raise the interfacial tension. ${ }^{38}$ While water-explicit simulations, in principle, account for hydration and non-ideal solution effects, the salt-induced lowering of the dielectric constant, and ion-surface interactions, provided that accurate force fields are used, the in-depth understanding of ion-specific effects and the description of experimental scenarios that go beyond idealized planar interfaces require the use of coarse-grained simulations and analytically tractable theories.

Besides simulations, there are several approximate methods that can be used to predict electrolyte chemical potentials. Starting with the pioneering work of Pitzer, ${ }^{39,40}$ various models were developed to describe the electrolyte activity by simple analytical expressions. ${ }^{41-43}$ The Mean Spherical Approximation (MSA) in fact very accurately predicts the salt solution activity compared to simulations using the same underlying Hamiltonian. ${ }^{44-46}$ It is well known that the dielectric constant of electrolyte solutions decreases with electrolyte concentration, ${ }^{47}$ and consequently, it was shown that in order to correctly predict the experimental activity of salt solutions at high concentrations, this dielectric decrement must be taken into account. ${ }^{48-53}$ Note that the dielectric decrement influences also the single-ion solvation free energy and thereby makes the solvation free energy dependent on the salt concentration. ${ }^{52,54}$ There are several models that describe the dielectric constant of salt solutions: ${ }^{5}$ the heuristic Gavish and Promislow model ${ }^{56}$ describes experimental data very accurately over the entire concentration range in terms of two fit parameters, namely, the infinite-dilution excess ionic polarizability and the molten-salt dielectric constant at zero water content. Using solvent-implicit simulations of interfacial ionic systems, it was seen that non-ideal solution effects as well as dielectric interfacial effects crucially influence the interfacial tension. ${ }^{57-59}$

In our work, we aim at integrating all these effects by the use of suitably constructed solvent-implicit simulation models and by modified Poisson-Boltzmann (MPB) approaches for monovalent electrolytes. As a simulation model, we use the so-called primitive model, where ions are described by hard charged spheres and water is described by a medium with a homogeneous dielectric constant.
TABLE I. Summary of the different MPB and MC models. For the MC models, the screened image-charge OS potential is implicitly included.

\begin{tabular}{llcc}
\hline \hline & OS & $\gamma_{ \pm}$ & $\varepsilon\left(c_{\mathrm{b}}\right)$ \\
\hline MPB-1 & No & Ideal & Constant \\
MPB-2 & Yes & Ideal & Constant \\
MPB-3 & Yes & Non-ideal & Constant \\
MPB-4 & Yes & Ideal & Depend \\
MPB-5 & Yes & Non-ideal & Depend \\
MC-2 & $\ldots$ & Ideal & Constant \\
MC-3 & $\ldots$ & Non-ideal & Constant \\
MC-4 & $\ldots$ & Ideal & Depend \\
MC-5 & $\ldots$ & Non-ideal & Depend \\
\hline \hline
\end{tabular}

We first compare experimental activity coefficient data for six different salts with grand-canonical Monte Carlo simulation (GCMC) results and with MSA results over the entire concentration range. The agreement between GCMC and MSA results is good for most concentrations, which is crucial because it allows us to use the closed-form MSA chemical potential expression for our subsequent calculation of interfacial tensions. The dielectric decrement of the salt solutions is in the simulations, and MPB approaches accounted for by the use of the Gavish and Promislow model, where the excess ionic polarizabilities are taken from experimental data, while the molten-salt dielectric constant parameters are obtained by fitting the experimental dielectric constant curves. The fit of the GCMC predictions to the experimental activity coefficient data yields in a second step the effective ionic diameters.

Using the salt-concentration-dependent bulk dielectric constants and the effective ionic diameters determined by comparison with experimental data, we then calculate excess surface tensions of salt solutions by means of Monte Carlo (MC) simulations that properly account for dielectric image-charge effects at the surface. The comparison with experimental data demonstrates that an additional ion-specific interaction between the air-water interface and the ions is present, which is more long-ranged than the ion radius and which is repulsive for all ions except iodide. Here, we also compare with a MPB model that accounts for image-charge effects [similar to the Onsager-Samaras (OS) theory ${ }^{60}$ ] but that also includes additional ion-specific ion-interface interactions. We end with a discussion of the relative importance of non-ideal solution effects, the dielectric decrement of salt solutions, and ion-interface interactions that include dielectric image-charge effects. Table I lists different MC and MPB models that are compared to each other.

\section{METHODS}

\section{A. Analytical model for activity coefficients}

The mean ionic chemical potential, averaged over cations and anions, can be written as

$$
\mu_{ \pm}\left(c_{\mathrm{b}}\right)=k_{\mathrm{B}} T \ln \left(d^{3} c_{\mathrm{b}} \gamma_{ \pm}\right)
$$

where $c_{\mathrm{b}}$ is the bulk salt concentration in terms of the number density of ion pairs, $k_{\mathrm{B}} T$ is the thermal energy, $d$ is the mean ionic diameter, and $\gamma_{ \pm}$is the mean ionic activity coefficient. Deviations of $\gamma_{ \pm}$from unity indicate non-ideal solution effects due to interactions 
between ions and between ions and water. ${ }^{52}$ For a system of charged hard spheres that interact via Coulomb interactions, the so-called primitive model, the mean ionic activity coefficient can be obtained by adding two contributions, the ion-ion term, modeled with MSA theory, ${ }^{44,48,57}$ and the ion-water contribution $\mu_{i w}$,

$$
\ln \gamma_{ \pm}=\frac{\kappa d \sqrt{(1+2 \kappa d)}-\kappa d-(\kappa d)^{2}}{24 \phi}+\frac{8 \phi-9 \phi^{2}+3 \phi^{3}}{(1-\phi)^{3}}+\beta \mu_{i w}
$$

where $\kappa=\sqrt{8 \pi q^{2} \ell_{\mathrm{B}}\left(c_{\mathrm{b}}\right) c_{\mathrm{b}}}$ is the inverse Debye length;

$$
\ell_{\mathrm{B}}\left(c_{\mathrm{b}}\right)=\frac{e^{2}}{4 \pi \varepsilon\left(c_{\mathrm{b}}\right) \varepsilon_{0} k_{\mathrm{B}} T}
$$

is the salt-concentration-dependent Bjerrum length; $e$ is the elementary charge; $q$ is the ion valency; $\varepsilon\left(c_{\mathrm{b}}\right)$ is the relative electrolyte dielectric constant, which depends on the bulk salt concentration and thereby makes the Bjerrum length and the Debye length saltconcentration dependent; $\varepsilon_{0}$ is the dielectric permittivity of vacuum; and $\phi=c_{\mathrm{b}} \pi d^{3} / 3$ is the ionic volume fraction. The Bjerrum length at infinite dilution $c_{\mathrm{b}}=0$ is set to $\ell_{\mathrm{B}}(0)=0.71 \mathrm{~nm}$, which is obtained using the pure water relative dielectric constant ${ }^{61} \mathcal{E}_{\mathrm{W}}=78.5$ at room temperature, $25^{\circ} \mathrm{C}$. The mean ionic activity coefficient depends on the ion type via the ionic diameter $d$, which is different for different ion combinations, and via the salt-concentration-dependent dielectric constant $\varepsilon\left(c_{\mathrm{b}}\right)$. The physical meaning of the mean ionic diameter $d$ is the minimal cation-anion separation at contact. This is so because the cation-anion interaction is attractive and therefore dominant compared to cation-cation and anion-anion interactions. The concentration-dependent ion-water contribution is obtained by the Born solvation model,

$$
\beta \mu_{i w}=\frac{e^{2}}{4 \pi \varepsilon_{0} k_{B} T a_{B}}\left(\frac{1}{\varepsilon\left(c_{\mathrm{b}}\right)}-\frac{1}{\varepsilon_{\mathrm{w}}}\right),
$$

where $\varepsilon_{\mathrm{w}}=\varepsilon\left(c_{\mathrm{b}}=0\right)$ denotes the pure water dielectric constant by which the ion-water contribution vanishes in the infinite-dilution limit. Here, $a_{B}$ is the ionic mean Born diameter, which is obtained from experimental data for the solvation free energy in the infinitedilution limit according to ${ }^{62}$

$$
\Delta G=\frac{e^{2}}{4 \pi \varepsilon_{0} a_{B}}\left(\frac{1}{\varepsilon_{\mathrm{w}}}-1\right) .
$$

Note that the ionic mean Born diameter $a_{B}$ is not necessarily the same as the mean ionic diameter $d$ that appears in the ion-ion interaction contribution to the activity coefficient. This reflects the fact that $a_{B}$ results from ion-water interactions and is modified due to non-linear dielectric effects, ${ }^{63}$ while $d$ reflects the closest distance between cations and anions. Fitting results for all diameters are shown in Table II. In Appendix A, we compare our fitting results with results for the $\mathrm{NaCl}$ solvation free energy obtained from all-atom MD simulations.

\section{B. Dielectric constant at finite salt concentration}

The dielectric constant of salt solutions decreases significantly with rising salt concentration in a fashion that depends on the salt type. The experimental ion-specific dielectric decrement can be very well described by the heuristic Gavish-Promislow model, ${ }^{56}$

$$
\varepsilon\left(c_{\mathrm{b}}\right)=\varepsilon_{\mathrm{w}}+\left(\varepsilon_{\mathrm{w}}-\varepsilon_{\mathrm{ms}}\right)\left[\frac{1}{\tanh \left(\frac{3 \alpha_{\mathrm{p}} c_{\mathrm{b}}}{\varepsilon_{\mathrm{w}}-\varepsilon_{\mathrm{ms}}}\right)}-\frac{\left(\varepsilon_{\mathrm{w}}-\varepsilon_{\mathrm{ms}}\right)}{3 \alpha_{\mathrm{p}} c_{\mathrm{b}}}\right] .
$$

In the limit of low concentration $c_{\mathrm{b}}$, one obtains $\varepsilon\left(c_{\mathrm{b}}\right) \simeq \varepsilon_{\mathrm{w}}+\alpha_{\mathrm{p}} c_{\mathrm{b}}$, while in the limit of high concentration, one obtains $\varepsilon\left(c_{\mathrm{b}}\right) \simeq \varepsilon_{\mathrm{ms}}$. The model contains two fitting parameters: the dielectric constant of the molten salt, denoted as $\varepsilon_{\mathrm{ms}}$, and the excess ionic polarizability per ion pair $\alpha_{\mathrm{p}}$ in the infinitely dilute limit. Here, we take the values of $\alpha_{\mathrm{p}}$ from experimental measurements, listed in Table II, so that we are left with only a single fit parameter $\varepsilon_{\mathrm{ms}}$ for each ion pair.

\section{Grand-canonical Monte Carlo simulations for bulk salt solutions}

To obtain the activity coefficient of a bulk salt solution as a function of salt concentration, we perform grand-canonical simulations and thereby measure the concentration as a function of the imposed chemical potential. The Born solvation part, given by Eq. (4), is added to the chemical potential imposed in the simulations. Besides regular MC moves, where single ions are moved in random directions, the GCMC method consists of insertions and removals of ion pairs that conserve the system electroneutrality, using the proper acceptance rules. ${ }^{64,65}$ In the present case, where the dielectric constant depends on the salt concentration, we update the dielectric constant, which enters the calculation of the system energy, every $10 \mathrm{MC}$ steps according to the mean ion concentration. For high salt concentrations, where the dielectric constants are low, we update the dielectric constant more frequently in order to accelerate equilibration.

The Coulomb energy is calculated using Ewald summation methods ${ }^{65}$ based on the energy expression

$$
U / k_{\mathrm{B}} T= \begin{cases}\ell_{\mathrm{B}}\left(c_{\mathrm{b}}\right) \sum_{j=1}^{N} \sum_{k=j+1}^{N} \frac{q_{j} q_{k}}{r_{j k}} & \text { for } r_{j k} \geq d \\ \infty & \text { for } r_{j k}<d\end{cases}
$$

TABLE II. Summary of electrolyte parameters. The ionic diameters $d$ are obtained by fits to the experimental activity coefficients in Fig. 3. The limiting dielectric constants at zero water content, $\varepsilon_{\mathrm{ms}}$, are obtained by fits to the experimental dielectric data in Fig. 2, while the excess ionic polarizabilities $\alpha_{p}$ are extracted from experimental data. ${ }^{47,78,81,82}$ The value of $\varepsilon_{\mathrm{ms}}$ for $\mathrm{NaF}$ is taken to be the same as KF because of the restricted experimental concentration range of the dielectric data for $\mathrm{NaF}$. The mean ionic Born diameters $a_{B}$ are obtained from the Born model in Eq. (5) and experimental data from Ref. 62.

\begin{tabular}{lcccc}
\hline \hline Salt & $d(\mathrm{~nm})$ & $\varepsilon_{\mathrm{ms}}$ & $\alpha_{\mathrm{p}}\left(\mathrm{M}^{-1}\right)$ & $a_{B}(\mathrm{~nm})$ \\
\hline $\mathrm{NaCl}$ & 0.249 & 27.9 & -11.59 & 0.369 \\
$\mathrm{KCl}$ & 0.23 & 35 & -10.02 & 0.406 \\
$\mathrm{NaI}$ & 0.27 & 18.3 & -14.58 & 0.392 \\
$\mathrm{KI}$ & 0.25 & 30.5 & -14.69 & 0.433 \\
$\mathrm{NaF}$ & 0.17 & 22.4 & -11.9 & 0.340 \\
$\mathrm{KF}$ & 0.24 & 22.4 & -12 & 0.371 \\
\hline \hline
\end{tabular}


where $r_{j k}$ is the distance between ions $j$ and $k$ of valencies $q_{j}$ and $q_{k}$, respectively, and $N$ is the fluctuating total number of particles. Typical particle numbers are between 500 and 1000 . The cubic simulation box size is between $L_{\mathrm{b}}=4 \mathrm{~nm}$ and $L_{\mathrm{b}}=25 \mathrm{~nm}$, depending on the bulk ion concentration.

\section{Canonical Monte Carlo simulations in slab geometry}

In the slab geometry, we perform canonical simulations at a fixed total ion number and estimate the bulk ion concentration from the concentration at the mid plane between the two surfaces, separated by distance $L$. The simulation model is schematically shown in Fig. 1. Cations and anions have the same diameter $d$, which is obtained from fits to experimental mean activity coefficient data $\gamma_{ \pm}$, as will be explained in Sec. III. Ions interact with the interfaces via a square-well box potential of strength $\alpha_{i}$ if they approach the interfaces closer than the distance $h_{ \pm}=0.5 \mathrm{~nm}$. The interface interaction parameters $\alpha_{i}$ are fitted by comparison with experimental interfacial tension data. The interaction range $h_{ \pm}=0.5 \mathrm{~nm}$, which is significantly larger than the ionic radii that are in the range of $d / 2$ $=0.08 \mathrm{~nm}-0.13 \mathrm{~nm}$, was previously shown to be in qualitative agreement with interface-ion interaction profiles extracted from waterexplicit molecular dynamics simulations and furthermore allows us to robustly fit experimental interfacial tension data. ${ }^{66}$ In one test simulation, we show interfacial tension results for the choice $h_{ \pm}$ $=d / 2$ [the green line in Fig. 4(a)] and demonstrate that this interaction range is too small and cannot be used to describe the experimental data.

The Coulomb energy for MC slab simulations includes direct ion-ion as well as ion-interface image-charge interactions. There are various methods for taking into account surface polarization effects due to the difference of the dielectric constants of the salt solution and the surrounding air. ${ }^{67-72}$ We choose the method developed in Ref. 73, which extends the modified Ewald sum and considers an infinite number of image charges. We therefore use the energy expression

$$
U / k_{\mathrm{B}} T=\left\{\begin{array}{l}
\ell_{\mathrm{B}}\left(c_{\mathrm{b}}\right) \sum_{j=1}^{N} \sum_{k=j+1}^{N} \frac{q_{j} q_{k}}{r_{j k}} \\
+\sum_{l=1}^{\infty} \frac{\chi\left(c_{\mathrm{b}}\right)^{l} \ell_{\mathrm{B}}\left(c_{\mathrm{b}}\right)}{2} \sum_{j=1}^{N} \sum_{k=1}^{N} \frac{q_{j} q_{k}}{r_{j k}^{\prime}} \\
+\sum_{l=1}^{\infty} \frac{\chi\left(c_{\mathrm{b}}\right)^{l} \ell_{\mathrm{B}}\left(c_{\mathrm{b}}\right)}{2} \sum_{j=1}^{N} \sum_{k=1}^{N} \frac{q_{j} q_{k}}{r_{j k}^{\prime \prime}} \\
+U_{s} / k_{\mathrm{B}} T \text { for } r_{j k} \geq d \\
\infty \text { for } r_{j k}<d, z_{j},<0 \text { or } z_{j}>L,
\end{array}\right.
$$

where $\chi\left(c_{\mathrm{b}}\right)=\left[\varepsilon\left(c_{\mathrm{b}}\right)-1\right] /\left[\varepsilon\left(c_{\mathrm{b}}\right)+1\right]$ is the dielectric contrast between the slab interior, which is filled with electrolyte, and the surrounding air. The surface energy $U_{s}$ is given by

$$
U_{s} / k_{\mathrm{B}} T=\sum_{j=1}^{N} \alpha_{i}(j)\left[1-\theta\left(z_{j}-h_{ \pm}\right)+\theta\left(z_{j}-L+h_{ \pm}\right)\right] .
$$

The function $\theta(z)$ is the Heaviside function, and the surface interaction strength $\alpha_{i}(j)$ for the $j$ th ion depends on ion type $i . N=1000$ is the total number of particles (500 cations and 500 anions), $r_{j k}$ is a)

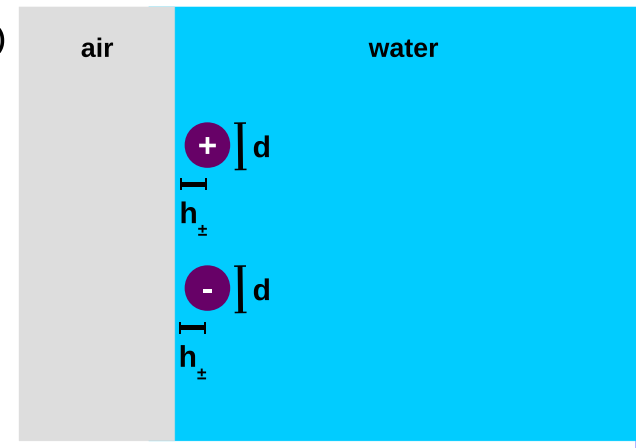

air

b)

c)

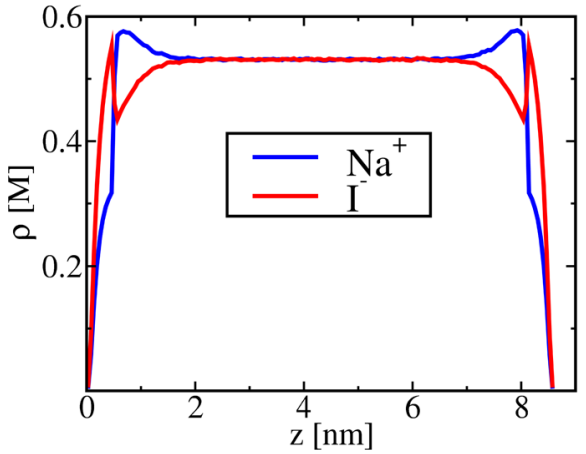

FIG. 1. Schematic geometry of the model for ions near air-electrolyte interfaces (a) In the electrolyte medium, the dielectric constant depends on the bulk ionic concentration, $\varepsilon\left(c_{b}\right)$, while in air, the dielectric constant is set to 1 . (b) The interface box potential accounts for ion-interface interactions that are due to interfacial hydration effects. (c) Exemplary ionic density profile for Nal at a bulk salt concentration of $c_{b}=0.53 \mathrm{M}$ from MC simulations using the MC- 5 model.

the distance between ions $j$ and $k$, and $r_{j k}^{\prime}$ and $r_{j k}^{\prime \prime}$ are the distances between charge $j$ and image charges $k$ located in regions $z<0$ and $z>L$, respectively. The simulation box widths are $L_{x}=L_{y}$ in the lateral directions (depending on the ionic concentration) and $L_{z}=20 L_{x}$ in the direction normal to the interfaces (see Refs. 73 and 74 for more details). The interface separation is chosen as $L=20 \kappa^{-1}$, where $\kappa$ is the inverse Debye length, in order to prevent interactions between the two interfaces. Thus, the Coulomb energy used in the MC simulations takes into account the dependency of the Bjerrum length and the electrolyte dielectric constant on the bulk ion concentration. 
The excess interfacial tension is determined by the Gibbs adsorption isotherm

$$
\Delta \sigma=-2 \int \Gamma_{ \pm} d \mu_{ \pm}
$$

where $\Gamma_{ \pm}$is the mean ionic excess per unit area, which follows from the ionic concentration profiles obtained in the MC simulations as

$$
\Gamma_{ \pm}=\frac{N}{4 L_{x}^{2}}-c_{\mathrm{b}} L / 2
$$

where $c_{\mathrm{b}}$ is the mean ionic bulk concentration far from the interfaces in the middle of the simulation box. Using for the non-ideal chemical potential Eq. (1), Eq. (10) can be straightforwardly integrated numerically.

\section{E. Modified Poisson-Boltzmann theory}

The MPB theory provides an analytical route to predict the interfacial tension of electrolytes including the effects of interfaceion interactions and dielectric decrement effects. For an interface located at $z=0$ and the electrolyte present at $z>0$, the short-ranged effective interaction potential $U_{i}(z)$ between an ion of type $i$ and the interface can be written as

$$
\frac{U_{i}(z)}{k_{\mathrm{B}} T}=\chi\left(c_{\mathrm{b}}\right) \frac{\mathrm{e}^{\kappa d}}{1+\kappa d} \frac{\ell_{\mathrm{B}}\left(c_{\mathrm{b}}\right)}{4 z} \mathrm{e}^{-2 \kappa z}+\alpha_{i} \theta\left(h_{ \pm}-z\right),
$$

where $\alpha_{i}$ is the ion-specific amplitude of the box potential, as in the MC simulations, and the first term reflects screened imagecharge interactions. ${ }^{75,76}$ The mean ion diameter $d$ and the box potential range $h_{ \pm}$are the same as in the MC simulations. The electrolyte dielectric constant depends on the ionic bulk concentration $c_{\mathrm{b}}$ according to Eq. (6), and thereby, $\chi, \kappa$, and $\ell_{\mathrm{B}}$ depend on $c_{\mathrm{b}}$ as well. In the limit when the dielectric constant is salt independent and the ionic diameter $d$ is taken to be zero, the first term in Eq. (12) reduces to the interface potential used in the original Onsager-Samaras (OS) theory. ${ }^{60}$

We include the ion-interface interaction potential into the MPB equation as

$$
\varepsilon\left(c_{\mathrm{b}}\right) \varepsilon_{0} \frac{d^{2} \psi}{d z^{2}}=-e c_{\mathrm{b}} \sum_{i} q_{i} \mathrm{e}^{-e q_{i} \psi(z) / k_{\mathrm{B}} T-U_{i}(z) / k_{\mathrm{B}} T} .
$$

Here, we neglect any dependence of $\varepsilon$ on the local ion concentration and consider it to be uniform.

The individual ionic surface excesses follow as

$$
\Gamma_{i}=c_{\mathrm{b}} \int_{0}^{\infty}\left(\mathrm{e}^{-e q_{i} \psi(z) / k_{\mathrm{B}} T-U_{i}(z) / k_{\mathrm{B}} T}-1\right) d z .
$$

Charge neutrality dictates $\Gamma_{+}=\Gamma_{-}$; we thus define the mean ionic surface excess $\Gamma_{ \pm}=\Gamma_{+}=\Gamma_{-}$. The Gibbs adsorption isotherm [Eq. (10)] can be written in terms of the activity coefficient as

$$
\Delta \sigma=-2 k_{\mathrm{B}} T \int_{0}^{c_{\mathrm{b}}} \Gamma_{ \pm}\left(1+c_{\mathrm{b}} \frac{d \ln \gamma_{ \pm}}{d c_{\mathrm{b}}}\right) \frac{d c_{\mathrm{b}}}{c_{\mathrm{b}}} .
$$

In the ideal limit, one has $c_{\mathrm{b}} d \ln \gamma_{ \pm} / d c_{\mathrm{b}}=0$, while in the general nonideal case, we obtain from Eq. (2),

$$
\begin{aligned}
c_{\mathrm{b}} \frac{d \ln \gamma_{ \pm}}{d c_{\mathrm{b}}}= & \frac{\kappa d \sqrt{1+2 \kappa d}-\kappa d(1+\kappa d)}{16 \pi c_{\mathrm{b}} d^{3} \sqrt{1+2 \kappa d}}+\frac{2 \phi(4-\phi)}{(1-\phi)^{4}} \\
& -\frac{1}{8 \pi d^{3}}\left[\sqrt{1+2 \kappa d}+\frac{\kappa d}{\sqrt{1+2 \kappa d}}-1-2 \kappa d\right] \frac{\kappa d}{2 \varepsilon} \frac{d \varepsilon}{d c_{\mathrm{b}}} \\
& -\frac{e^{2}}{4 \pi \varepsilon_{0} k_{\mathrm{B}} T a_{\mathrm{B}}} \frac{c_{\mathrm{b}}}{\varepsilon^{2}} \frac{d \varepsilon}{d c_{\mathrm{b}}},
\end{aligned}
$$

where we obtain from Eq. (6) the result

$$
\frac{d \varepsilon}{d c_{\mathrm{b}}}=\frac{\left(\varepsilon_{\mathrm{w}}-\varepsilon_{\mathrm{ms}}\right)^{2}}{3 \alpha_{\mathrm{p}} c_{\mathrm{b}}^{2}}-\frac{3 \alpha_{\mathrm{p}}}{\left[\sinh \left(\frac{3 \alpha_{\mathrm{p}} c_{\mathrm{b}}}{\varepsilon_{\mathrm{w}}-\varepsilon_{\mathrm{ms}}}\right)\right]^{2}} .
$$

To discuss the relative importance of non-ideal solution, ioncorrelation, and salt-concentration-dependent dielectric effects, we consider five different MPB models and four different MC models, in which we separately include or neglect the non-ideal chemical potential, the concentration dependence of $\varepsilon$, and, for the MPB model only, the OS potential [the first term in Eq. (12)]. The different models are summarized in Table I. For the MPB-1 model, an analytic solution is available. ${ }^{66}$ The MPB- 2 model is identical to the Onsager-Samaras theory in the limit of $\kappa d \rightarrow 0$ and $\alpha_{i} \rightarrow 0$, in which limit the surface tension can be exactly calculated as ${ }^{60}$

$$
\Delta \sigma=-\frac{2 \pi k_{\mathrm{B}} T}{\ell_{\mathrm{B}}^{2}} \sum_{n=1}^{\infty} \frac{n^{n}}{n ! n !} \frac{h^{n+1}}{n+1}\left(\ln h+g_{n}\right),
$$

where $g_{n}=\ln n+1+2 \gamma-1 /(n+1)-\sum_{m=1}^{n}(1 / m), h=\chi \kappa \ell_{\mathrm{B}} / 2$, and $\gamma$ is the Euler constant. A comparison of the Onsager-Samaras theory with MPB modeling results is shown in Fig. 10 in Appendix B. For models MPB-2 to MPB-5, we solve Eq. (13) by the shooting method and then integrate Eq. (15) using the trapezoidal rule with integration steps of $\Delta c_{\mathrm{b}}=10 \mathrm{mM}$.

\section{RESULTS}

\section{A. Bulk effects}

In Fig. 2, we compare experimental dielectric constant data with the heuristic model [Eq. (6)]. The values for the excess ionic polarizabilities, $\alpha_{\mathrm{p}}$, are directly taken from experiments, where we average the values given in Refs. 47, 78, 81, and 82 (see Table II). The resultant fit values for $\varepsilon_{m s}$ are given in Table II. The fit of $\varepsilon_{\mathrm{ms}}$ for $\mathrm{NaF}$ is not conclusive because the maximum solubility is around $1 \mathrm{M}$, and thus, the available data range is very small; we therefore use for $\mathrm{NaF}$ the same $\varepsilon_{m s}$ value as determined for KF.

In Fig. 3, we compare experimentally measured activity coefficients for various salts ${ }^{83}$ (filled symbols) with GCMC simulation results (open symbols) as well as with MSA theory (solid and broken lines). In both theoretical models, we include the Born solvation contribution $\beta \mu_{i w}$ from Eq. (4). In Fig. 3(a), we show results for the sodium salts, and in Fig. 3(b), we present results for the potassium salts. Note the different ordering of the salts with respect to the anions: in Fig. 3(a), the expected ordering iodide-chloride-fluoride (from top to bottom) is obtained, while in Fig. 3(b), the ordering 

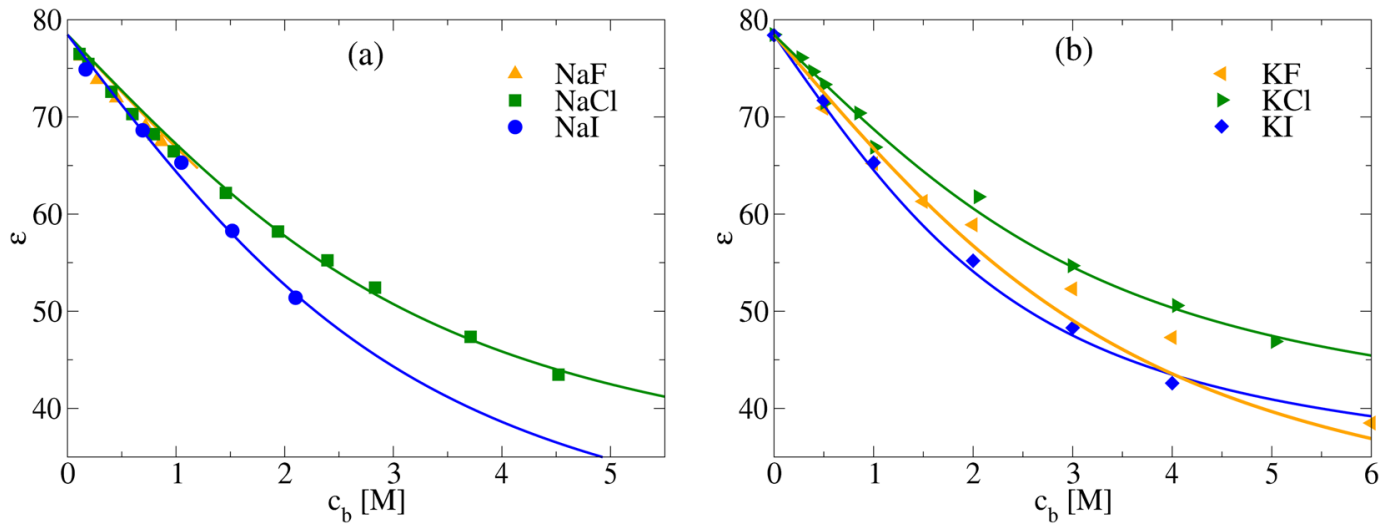

FIG. 2. Dielectric constants as a function of salt concentration. Symbols are experimental data, ${ }^{55,77-80}$ and lines correspond to the Gavish-Promislow model [Eq. (6)] with parameters determined by a non-linear fitting procedure (see Table II). (a) Data for $\mathrm{NaF}, \mathrm{NaCl}$, and $\mathrm{Nal}$. (b) Data for KF, $\mathrm{KCl}$, and $\mathrm{KI}$.

is partially reversed. Such ion-specific series reversals are well documented in the literature, ${ }^{52,86}$ have their origin in nonlinear ionic hydration effects, and occur for all different kinds of experimental observables. $^{8}$

The only remaining fitting parameter in the modeling is the ionic diameter $d$, which we determine by a fit of the GCMC results to the experimental data; for the MSA predictions, we use the same values of $d$. It is seen that the MSA model agrees well with GCMC and reproduces the experimental activity coefficients in a satisfactory fashion over the entire available concentration range. The broken lines show MSA theory predictions for the case where the dielectric constant is fixed at the pure water value and the ionic diameters are the same as used for the solid lines. The difference between these two MSA predictions is immense, which illustrates the importance of taking the salt-induced modifications of the dielectric constant into account.
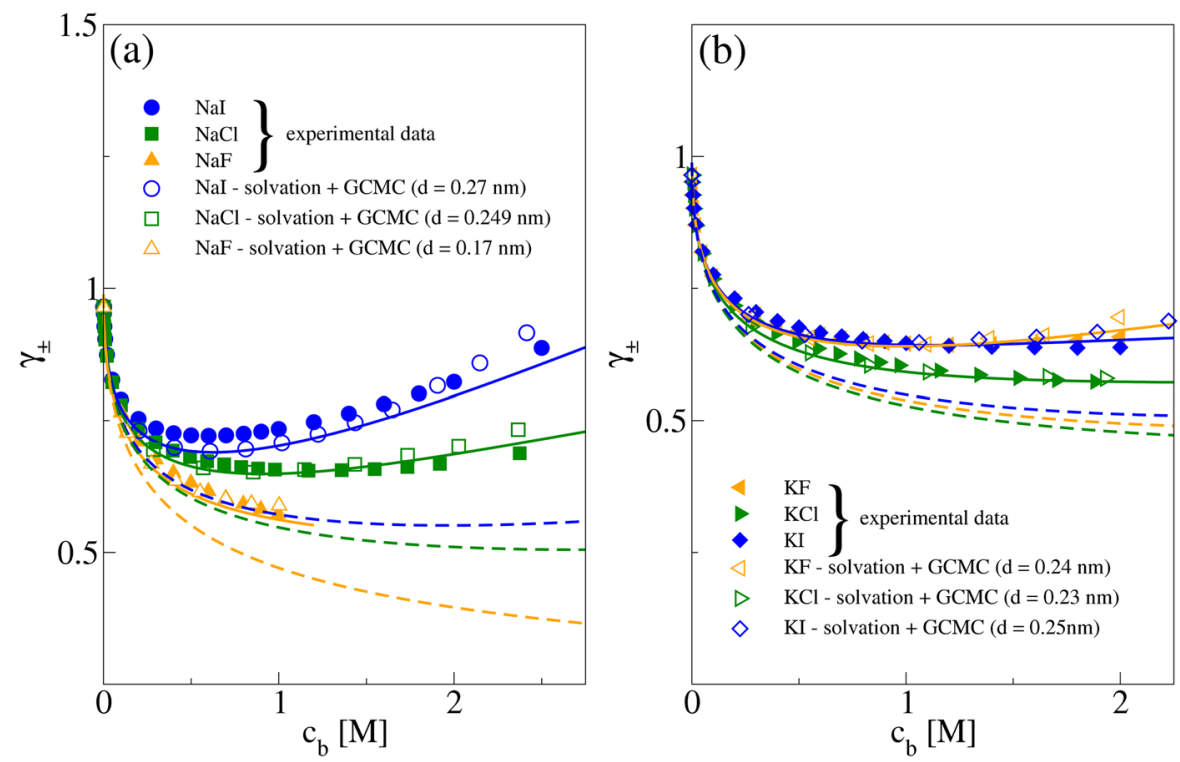

FIG. 3. Mean ionic activity coefficient as a function of salt concentration for (a) $\mathrm{NaF}, \mathrm{NaCl}$, and $\mathrm{Nal}$, and (b) $\mathrm{KF}, \mathrm{KCl}$, and $\mathrm{KI}$. The experimental data are represented by full symbols. ${ }^{83}$ The empty symbols represent the GCMC simulations combined with the Born solvation model including the salt-concentration-dependent dielectric function, from which the ion diameters $d$ are obtained by fits to the experimental data. The solid lines represent MSA theory [Eq. (2)] using the same ionic diameters, dielectric molten-salt parameters $\varepsilon_{\mathrm{ms}}$, and Born solvation contribution. The dashed lines represent MSA theory for the same ion diameters $d$ but for fixed dielectric constant corresponding to pure water. The units were transformed from molality to molarity using experimental solution mass density data for $\mathrm{KCl}{ }^{84}$ and $\mathrm{NaCl} .{ }^{85} \mathrm{As}$ the correction is negligible for $\mathrm{KCl}$ and $\mathrm{NaCl}$, we assume the same to be true also for the other salts (for which no data are available). 
The parameters obtained for all salts are summarized in Table II. The agreement between simulation results and MSA theory is satisfactory; we will therefore use the MSA result for the activity coefficient [Eq. (2)] when needed later to calculate the tension of the electrolyte-air interface according to Eq. (10). The deviations observed between MSA and simulations for higher concentrations, as seen for NaI in Fig. 3(a), can be explained by the low dielectric constant of $\mathrm{NaI}$ solutions at high concentrations, which leads to a high electrostatic coupling constant, as is explained in Appendix C. Interestingly, we note that when one neglects the ion solvation contribution to the activity coefficient, the agreement between experimental data and model prediction looks better, while the fitted diameters increase significantly, as discussed in Appendix D (Fig. 12).

The fit results for the ionic diameter $d$ demonstrate that there is no unique way to split the ionic diameters into individual ionic radii. Consider, for example, the fitted diameters $d$ for $\mathrm{NaCl}$ and $\mathrm{KCl}$ (see Table II). For the salts involving $\mathrm{Cl}^{-}$, the $\mathrm{Na}^{+}$radius would be higher than the $\mathrm{K}^{+}$radius, while for the salts involving $\mathrm{F}^{-}$, the opposite trend is obtained and the $\mathrm{K}^{+}$radius is larger than the $\mathrm{Na}^{+}$ radius. As mentioned before, this ion specific reversal of effective ionic radii is well-known in the literature. ${ }^{52,88}$ The large difference between the ionic Born diameters $a_{B}$ and the interaction ionic diameters $d$ as extracted from fits of the experimental activity coefficients is in Appendix A discussed in the context of non-linear dielectric effects, which are extracted from water-explicit all-atom molecular dynamics simulations. Since the solvation even of monovalent ions involves considerable non-linear dielectric contributions, the Born diameter $a_{B}$ has only limited physical meaning.

\section{B. Interfacial effects}

We now turn to interfacial effects. We calculate the excess surface tension by a combination of MC simulations and MSA theory, where we integrate Eq. (10) based on ion density profiles from MC simulations, using for the non-ideal chemical potential the MSA expression [Eq. (1)]. We first consider the MC-5 model that includes non-ideal bulk as well as concentration-dependent dielectric effects using parameters that were obtained from fits to experimental activity coefficients (see Table II). We first neglect ion-interface interactions and set $\alpha_{\mathrm{Na}}=\alpha_{\mathrm{Cl}}=\alpha_{\mathrm{I}}=0$. The MC simulation results are represented by red solid lines in Figs. 4(a) and 4(b) and compared with experimental data from different sources. The simulation results significantly underestimate the experimental data for $\mathrm{NaCl}$ in (a). The green line in Fig. 4(a) shows simulation results where a steric (i.e., infinitely high) ion-surface repulsion is considered, and we use $d / 2=0.1245 \mathrm{~nm}$ as the distance of closest approach for $\mathrm{Na}^{+}$as well as $\mathrm{Cl}^{-}$ions. It is seen that steric repulsion from the
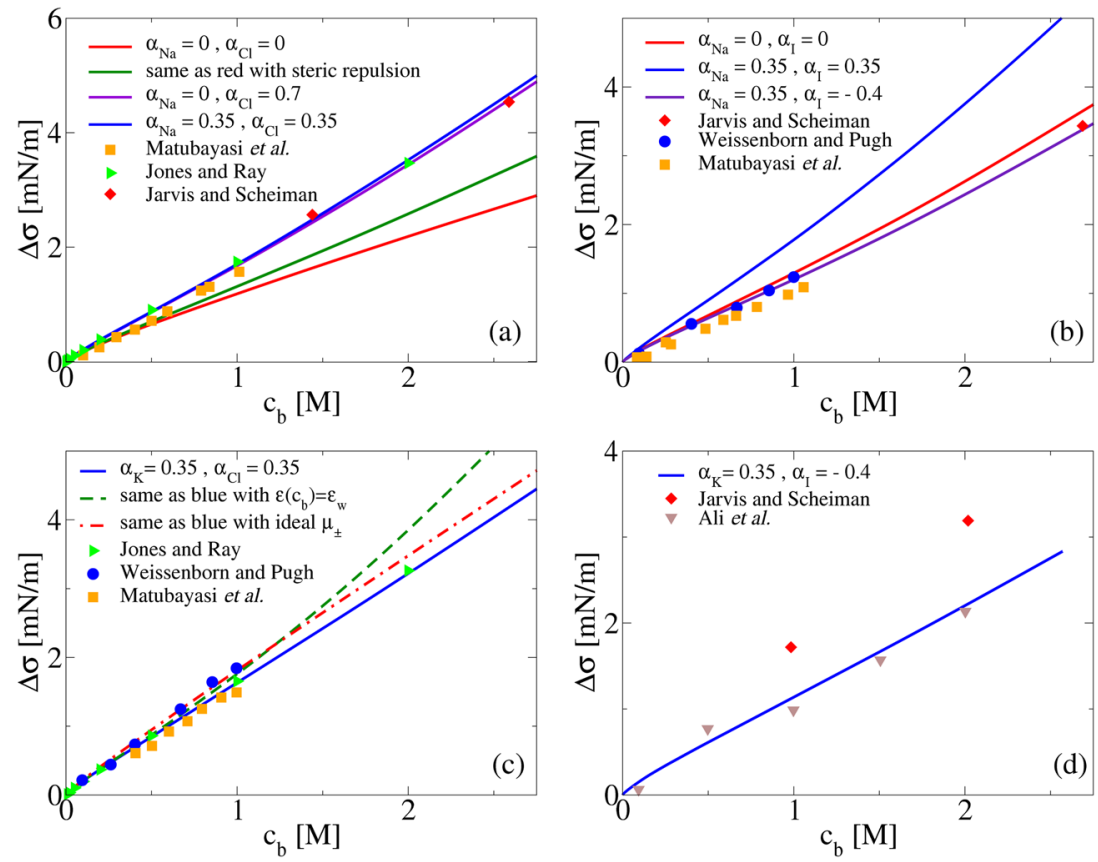

FIG. 4. Comparison of experimental and MC simulation excess interfacial tension data. (a) NaCl: squares, right triangles, and diamonds represent the experimental data of Matubayasi et al., ${ }^{6}$ Jones and Ray, ${ }^{2}$ and Jarvis and Scheiman, ${ }^{3}$ respectively. (b) Nal: squares, diamonds, and circles represent the experimental data of Matubayasi et al., Jarvis and Scheiman, ${ }^{3}$ and Weissenborn and Pugh, ${ }^{4}$ respectively. (c) $\mathrm{KCl}$ : squares, right triangles, and circles represent the experimental data of Matubayasi et al., Jones and Ray, ${ }^{2}$ and Weissenborn and Pugh, ${ }^{4}$ respectively. (d) Kl: down triangles and diamonds represent the experimental data of Ali et al. ${ }^{89}$ and Jarvis and Scheiman, respectively. Solid lines denote MC simulation results using the MC-5 model. The dashed line in (c) represents MC simulation results using the MC-3 model, where the dielectric constant equals pure water while a non-ideal chemical potential is used. The dotted-dashed line in (c) denotes simulations using the MC-4 model where an ideal chemical potential is used, but a concentration-dependent dielectric constant is employed. 


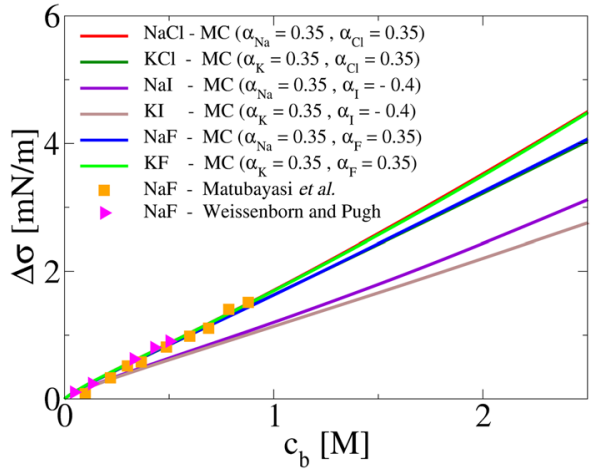

FIG. 5. Comparison of experimental interfacial tension data for $\mathrm{NaF}^{4}$ and $\mathrm{MC}$ simulation results using the MC- 5 model for all salt types considered in this work.

interface with a range given by the ionic radius $d / 2$ is not sufficient to correctly account for the $\mathrm{NaCl}$ experimental data. The violet curve represents simulation results where we use an ion-interface interaction range of $h_{ \pm}=0.5 \mathrm{~nm}$ (as explained in Sec. II) and obtain by fitting $\alpha_{\mathrm{Cl}}=0.7$ while fixing $\alpha_{\mathrm{Na}}=0$. Alternatively, the blue curve is the best fit when we enforce $\alpha_{\mathrm{Na}}=\alpha_{\mathrm{Cl}}$, in which case we obtain $\alpha_{\mathrm{Na}}=0.35$ and $\alpha_{\mathrm{Cl}}=0.35$. It is seen that both parameter sets reproduce very well the experimental data. In consistency with our previous modeling results ${ }^{66}$ and with our previous molecular dynamics simulation results, ${ }^{37}$ we choose the latter parameter set where sodium and chloride are equally repelled from the interface for our further calculations. Note that this repulsion acts on top of the dielectric image-charge repulsion, which is included explicitly in the MC simulations.

By fixing $\alpha_{\mathrm{Na}}=0.35$, we obtain $\alpha_{\mathrm{I}}=-0.4$ by a fit of our simulation results (purple curve) to the experimental data of $\mathrm{NaI}$ in Fig. 4(b). Here, it is seen that choosing $\alpha_{\mathrm{Na}}=0.35$ and $\alpha_{\mathrm{I}}=0.35$ (blue curve) overestimates the experimental data, while choosing
TABLE III. Summary of the interfacial affinity parameters $\alpha_{i}$ obtained from the fits in Figs. 4 and 6 .

\begin{tabular}{lllllr}
\hline \hline & $\mathrm{Na}$ & $\mathrm{K}$ & $\mathrm{Cl}$ & $\mathrm{F}$ & $\mathrm{I}$ \\
\hline MC-5 & 0.35 & 0.35 & 0.35 & 0.35 & -0.4 \\
MPB-1 & 1.2 & 0.9 & 1.2 & 0.9 & 0.3 \\
MPB-5 & 0.8 & 0.8 & 0.8 & 0.9 & -0.4 \\
\hline \hline
\end{tabular}

$\alpha_{\mathrm{Na}}=0$ and $\alpha_{\mathrm{I}}=0$ (red curve) describes the experimental data quite well. We thus conclude that the iodide ion is slightly attracted to the air-electrolyte interface with a strength that amounts to roughly a third of the thermal energy, in agreement with previous simulation reports ${ }^{28,31}$ and experiments. ${ }^{90}$ For the $\mathrm{KCl}$ and KI data in Figs. 4(c) and 4 (d), we fix $\alpha_{\mathrm{Cl}}=0.35$ and $\alpha_{\mathrm{I}}=-0.4$ and choose $\alpha_{\mathrm{K}}=0.35$, which gives a reasonably good comparison with the experimental data. We note that because of the considerable scattering between the experimental data from different sources, a fit of the experimental data is not meaningful.

In Fig. 5, we compare experimental data for $\mathrm{NaF}$ with $\mathrm{MC}$ results for which we choose $\alpha_{\mathrm{F}}=0.35$, and here, a fit is not meaningful as the experimental data extend only to $1 \mathrm{M}$. In Fig. 5, we also include a summary of the MC results for all different salts considered in this paper, including KF (for which no experimental data are available) using the previously obtained parameters $\alpha_{\mathrm{K}}=0.35$ and $\alpha_{\mathrm{F}}=0.35$. The difference in the excess interfacial tension data between different salts is significant at high concentrations, and we note that these differences are caused by the combination of ion-interfacial interactions as well as dielectric and non-ideal effects. We conclude this section by noting that sodium, potassium, fluoride, and chloride are equally repelled from the interface, while the larger iodide ion is slightly attracted to the interface. These ion-interface interactions do not primarily reflect dielectric image effects, since these are included in the MC simulations, rather they stem from ion-specific interfacial hydration effects that
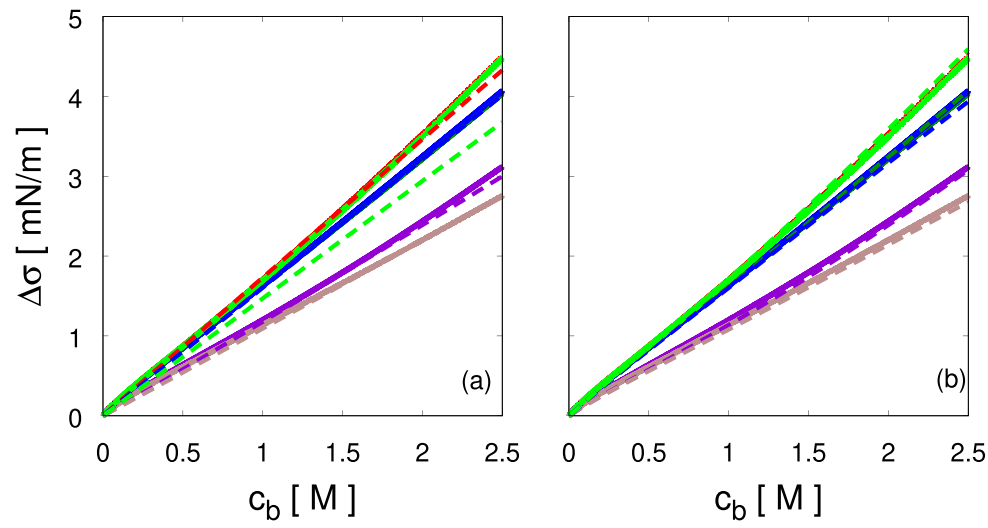

MC MPB

$$
\begin{array}{r}
\mathrm{NaCl}-= \\
\mathrm{KCl}-= \\
\mathrm{Nal}-= \\
\mathrm{Kl}-=- \\
\mathrm{NaF}-=- \\
\mathrm{KF}-=-
\end{array}
$$

FIG. 6. Comparison of the excess surface tension between MC simulations using the MC-5 model and two different MPB model results. Solid lines correspond to MC simulation results, whereas dashed lines correspond to MPB results. (a) MPB-1 model results are shown that neglect the image-charge OS potential, the non-ideal activity, and the concentration-dependent dielectric constant. (b) MPB-5 model results are shown that include the OS potential, the non-ideal activity, and the concentration-depending dielectric constant. The fitted affinity parameters $\alpha_{i}$ of the two different MPB models are summarized in Table III. For all models, we use $\varepsilon_{\mathrm{W}}=78.5$, $T=298 \mathrm{~K}$, and $h_{ \pm}=0.5 \mathrm{~nm}$ as well as $d, \alpha_{\mathrm{p}}$, and $\varepsilon_{\mathrm{ms}}$, as obtained in Sec. II A. 
go beyond the solvent-implicit point-like dielectric ion model. The important message here is that these effects can be accurately accounted for by adding short-ranged ion-interface interaction potentials into the MC simulations with interaction strength parameters that are obtained from fits to experimental interfacial tension data.

\section{Comparison of MC simulations and modified PB models}

So far, we compared MC simulations combined with MSA theory to experimental data in bulk and at interfaces. A very successful and standard model to predict the behavior of ions at interfaces is the Poisson-Boltzmann theory, which neglects correlations between ions but otherwise treats the long-ranged electrostatic interactions between ions exactly and has been very successfully applied to all kinds of different systems. ${ }^{91}$ At planar interfaces, the main shortcoming of $\mathrm{PB}$ approaches is that they neglect ion fluctuation effects but also the image-charge repulsion of ions from low-dielectric surfaces. This is so because the PB approach treats a smeared-out homogeneous ion cloud that does not produce an electric field that penetrates into the substrate. We here modify the PB approach such as to include the dielectric and other interfacial effects explicitly and compare this MPB model with our MC simulations. As explained in Sec. II, the MPB model includes image-charge repulsion via a heuristically added screened image-charge potential and is similar in spirit to the original Onsager-Samaras theory.

In Fig. 6, we compare MPB theory (broken lines) with MC simulation results (solid lines): in Fig. 6(a), we use the version MPB-1 that neglects the dielectric OS potential, non-ideal solution, and dielectric decrement effects, and in Fig. 6(b), we use the version MPB-5 that accounts for all these effects. Note that the MC data come from model MC-5 that includes non-ideal solution and dielectric decrement effects.

Our fitting strategy of the ion-interface interaction strengths $\alpha_{i}$ in the two MPB models is the same as used previously when we fitted the MC simulations to the experimental data. First, we fit $\alpha_{\mathrm{Na}}$ and $\alpha_{\mathrm{Cl}}$ for $\alpha_{\mathrm{Na}}=\alpha_{\mathrm{Cl}}$ to the $\mathrm{MC}$ data for $\mathrm{NaCl}$ (red line in Fig. 6). Then, we fit $\alpha_{\mathrm{K}}$ by comparison with the $\mathrm{MC}$ data for $\mathrm{KCl}$ (dark green line in Fig. 6), and after that, we fit $\alpha_{\mathrm{I}}$ by comparison with the MC data for $\mathrm{NaI}$ (purple line in Fig. 6). Finally, we fit $\alpha_{\mathrm{F}}$ by comparison with the MC data for NaF (blue line in Fig. 6). All other parameters such as $d, \alpha_{\mathrm{p}}$, and $\varepsilon_{\mathrm{ms}}$ are taken as obtained in Sec. II A. We find fair agreement between the MC and MPB-1 results in Fig. 6(a), and the agreement with the MPB-5 results in Fig. 6(b) is in fact very good.

The resulting fit values for $\alpha_{i}$ are summarized in Table III. We observe that the MPB-1 $\alpha_{i}$ values are significantly larger than the MC-5 values, which is easily explained by the fact that the MPB1 model does not include image-charge repulsion effects. To make up for this, the image-charge repulsion is accounted for by the box potential, which increases the interaction parameter. Note that the agreement between the MPB- 1 model and the MC- 5 simulation predictions for the interfacial tension in Fig. 6(a) is not bad, which indicates that the MPB-1 model, which is analytically solvable, is quite useful and accurate in practice. The $\alpha_{i}$ fit values for the MPB5 model are significantly reduced compared to the MPB-1 model, which is understandable because now the image-charge repulsion is, according to Eq. (12), taken into account explicitly, but we still note a significant difference from the MC-5 fit results. We attribute the reason for this to the fact that the screened image-charge potential in Eq. (12) underestimates the actual strength of the ionic image-charge repulsion from the interface due to the effects of ion correlations. For $\mathrm{NaCl}$ solutions, we obtain $\alpha_{\mathrm{Na}}=\alpha_{\mathrm{Cl}}=1.2$ using the MPB-1 model, which is slightly larger than the fit values obtained previously using the same model by comparison with molecular dynamics simulation results, in which case $\alpha_{\mathrm{Na}}=1.16$ and $\alpha_{\mathrm{Cl}}=0.98$ were obtained. ${ }^{66}$ We note that even though we use the same $\alpha_{i}$ values for $\mathrm{NaCl}$ and $\mathrm{KCl}$ in the MPB-5 model, the predicted surface tension curves differ from each other, similar to the MC-5 results. The reason for this is that the values for the ion diameter $d$, the Born diameter $a_{B}$, and the dielectric parameters $\alpha_{\mathrm{p}}$ and $\varepsilon_{\mathrm{ms}}$ for $\mathrm{NaCl}$ and $\mathrm{KCl}$ slightly differ, as summarized in Table II.

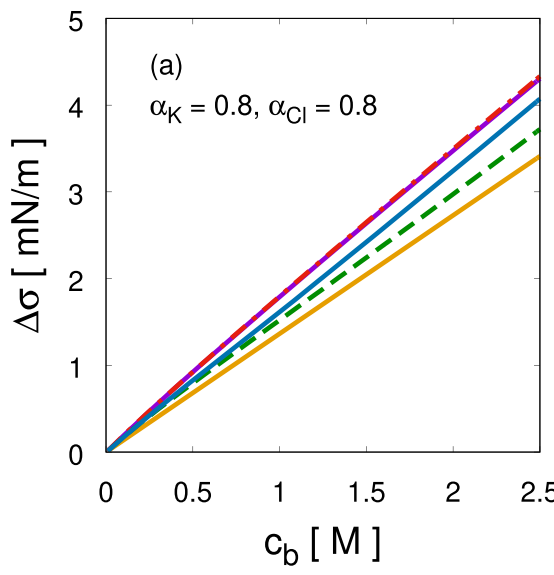

MPB-1 MPB-4 - MPB-2 - MPB-5 MPB-3 - -

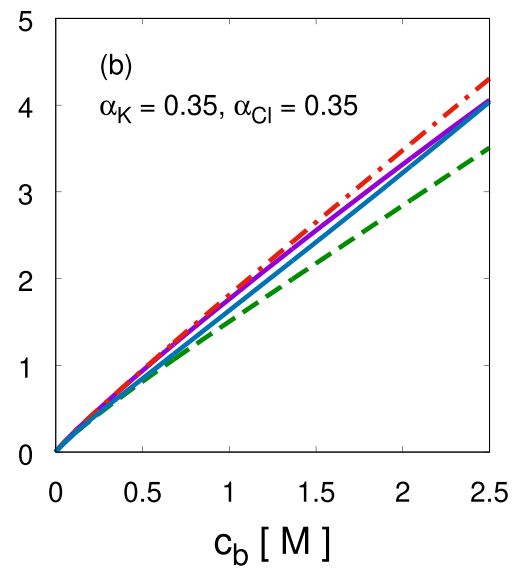

MC-2 - MC-4 - :-

MC-3 - - MC-5
FIG. 7. Decomposition of the effects of the image-charge OS potential, the non-ideal activity, and the concentrationdependent dielectric constant on the interfacial tension. (a) Comparison of all five MPB models. We use $\alpha_{\mathrm{K}}=\alpha_{\mathrm{Cl}}$ $=0.8$ with corresponding $d, \alpha_{p}$, and $\varepsilon_{\mathrm{ms}}$ as appropriate for $\mathrm{KCl}$. (b) Comparison of all four MC models. We use $\alpha_{\mathrm{K}}=\alpha_{\mathrm{Cl}}$ $=0.35$ with corresponding $d, \alpha_{\mathrm{p}}$, and $\varepsilon_{\mathrm{ms}}$ as appropriate for $\mathrm{KCl}$. 


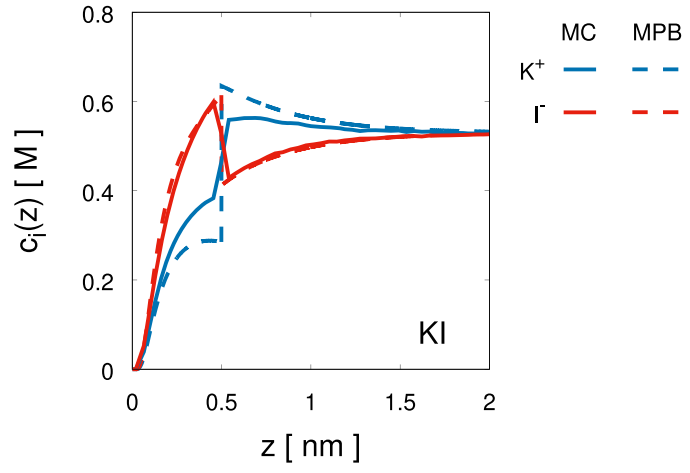

FIG. 8. Ion concentration profiles for a $0.53 \mathrm{M} \mathrm{KI}$ solution. The blue and red solid lines are the $\mathrm{K}^{+}$and $\mathrm{I}^{-}$concentrations calculated using the MC-5 model, whereas the blue and red dashed lines are calculated using the MPB- 5 model.

In the following, we decompose the effects of the OS screened image-charge potential, the non-ideal bulk activity, and the saltconcentration dependence of the dielectric constant on the interfacial tension, and we do this separately for the MC simulations and for the MPB model. Figure 7(a) shows the interfacial tension calculated from all five different MPB models for fixed $\alpha_{\mathrm{K}}=\alpha_{\mathrm{Cl}}=0.8$. For the MPB-1 model, the result (orange solid line) is strictly linear in the salt concentration and is below all other MPB model results because MPB-1 does not include the ion-interface dielectric repulsion as modeled by the OS potential. The results of the MPB-2 and MPB4 models, which both include the OS potential but neglect non-ideal solution effects, are almost the same, meaning that the bare effect of the concentration-dependent dielectric constant (i.e., in the absence of non-ideal solution effects) is minor. The result of MPB-3 has a lower slope than the result of MPB-2, demonstrating the effect of the non-ideal chemical potential. However, this decrease in slope is partly canceled when including in addition the concentrationdependent dielectric constant, as demonstrated by comparison of the MPB-5 result with the MPB-3 result. We conclude that ioninterface dielectric repulsion increases the interfacial tension, while non-ideal solution effects and salt-concentration-dependent dielectric effects partly cancel each other. The comparison of the four different MC models with $\alpha_{\mathrm{K}}=\alpha_{\mathrm{Cl}}=0.35$ in Fig. 7 (b) exhibits very similar trends.

In Fig. 8, we compare the ion density profiles obtained from MC-5 simulations and the MPB-5 model. We show profiles for a KI solution of bulk salt concentration $c_{\mathrm{b}}=0.53 \mathrm{M}$, where in the simulations the bulk salt concentration is determined from the concentration values far away from the interfaces. The MPB- 5 model profiles agree quite accurately with the MC simulation profiles, with some differences seen for the $\mathrm{K}^{+}$profile near $z=h_{ \pm}$, where the box potential produces a discontinuity, which is more drastic for the MPB-5 model since the amplitude $\alpha_{\mathrm{K}}$ is higher, as shown in Table III. We conclude that the MPB-5 model not only predicts interfacial tension data accurately but also gives a good account of the ion density profiles.

\section{CONCLUSIONS}

The excess air-water interfacial tensions of the six salt solutions $\mathrm{NaF}, \mathrm{NaCl}, \mathrm{NaI}, \mathrm{KF}, \mathrm{KCl}$, and $\mathrm{KI}$ are calculated by means of solvent-implicit MC simulations that model the ions as charged hard spheres. The MC simulations include correlation and fluctuation effects and account for dielectric image-charge effects that stem from the dielectric contrast between air and aqueous salt solutions. The effective ionic interaction diameters $d$ are obtained by fits of simulated salt solution activity coefficients to experimental data. This comparison also demonstrates that the dielectric decrement of salt solutions crucially influences the activities at high concentration and can be quantitatively described by a heuristic model with only two parameters that are extracted from experimental data.

In solvent-implicit models, the ion radius is an effective parameter that accounts for ion-specific bulk hydration effects. This effective ion radius is not directly related to the ion crystal radius. This is most vividly demonstrated by the ion specific reversal seen in the experimental bulk activity coefficient data in Fig. 3: in conjunction with sodium as a counterion, the ion radius increases from fluoride over chloride to iodide, as one would expect; in conjunction with potassium as a counterion, however, the ion radius decreases from fluoride to chloride.

By comparison of simulated and experimental interfacial tension data, we demonstrate that besides the dielectric decrement, non-ideal solution effects, and image-charge interfacial repulsion, we need to include an ion-specific ion-interface interaction, which has a range that is significantly larger than the ion diameter and which we fix at $0.5 \mathrm{~nm}$, guided by ion-interface interaction profiles previously extracted from water-explicit molecular dynamics simulations. The ion-interface interaction parameters for $\mathrm{Na}^{+}, \mathrm{K}^{+}, \mathrm{Cl}^{-}$, and $\mathrm{F}^{-}$are repulsive and given by $\alpha=0.35$ in units of $k_{\mathrm{B}} T$, while for $\mathrm{I}^{-}$, the interaction is attractive and given by $\alpha=-0.4$. In the interfacial MC simulations, the bulk chemical potential is needed for calculating the interfacial tension from the interfacial ionic excesses. For this, the chemical potential expressions from MSA theory are used, which are validated by bulk MC simulations.

In order to construct an even more coarse-grained description of ion-specific interfacial effects, we construct a mean-field MPB model that besides non-ideal solution and dielectric decrement effects also includes ion-interface interactions. The dielectric imagecharge repulsion of an ion from the low-dielectric air half space, which is missed in mean-field models at planar interfaces, is heuristically added to the ion potential by a screened image-charge potential, very much in the spirit of the original Onsager-Samaras theory. The strength of the ion-interface interaction potential is then obtained by comparison of the MPB model with the MC simulation results. MC and MPB predictions for interfacial tensions and ionic density profiles agree quite nicely, while the effective ion-interface interaction in the MPB models tends to be more repulsive by not more than $0.5 k_{\mathrm{B}} T$, which presumably comes from the neglect of correlation effects in MPB theory. By separately including and excluding the effects of non-ideal electrolyte activity and the concentrationdependent dielectric decrement, we show that these two effects are not additive and partially cancel each other.

Our MPB theory is a versatile, cheap, and quite accurate way of modeling ion-specific effects at interfaces, which accounts for dielectric image-charge effects by a heuristic potential. The comparison with MC simulation results demonstrates that the neglect of correlation effects, which is inherent to meanfield theories, is compensated by a slight shift of the effective 
ion-interface interaction potential strength. This explains why such MPB models with suitably adjusted effective ion-surface interaction potentials are able to describe experimental features very well. ${ }^{37,87}$ The minimal MPB model to consistently describe ion-specific effects in bulk and at interfaces thus needs to include the following features: ion-type dependent chemical potentials (which are accurately described by the closed-form MSA expression), ion-type dependent dielectric decrements (which are accurately described by the Gavish-Promislow model), as well as short ranged and ion-type dependent ion-interface potentials, with strengths that are adjusted so as to account for dielectric image-charge effects as well as for interfacial hydration effects.

\section{ACKNOWLEDGMENTS}

This work was supported by the Brazilian Research Council (CNPq) via Grant No. 302720/2018-9, FAPERGS, the Alexander von Humboldt Foundation through the CAPES program, the MaxPlanck Water Initiative, and the Deutsche Forschungsgemeinschaft (DFG) via Grant No. NE 810/11. Y.U. was supported by JSPS KAKENHI, Grant No. 20K14430. R.R.N. acknowledges support from the Infosys Foundation.

\section{APPENDIX A: ALL ATOM MOLECULAR DYNAMICS SIMULATIONS WITH EXPLICIT WATER: SINGLE-ION SOLVATION FREE ENERGIES}

Here, we calculate the solvation free energy $F$ of single $\mathrm{Na}^{+}$ and $\mathrm{Cl}^{-}$ions by thermodynamic integration using all-atom molecular dynamics simulations. The ion Lennard-Jones (LJ) parameters are taken from Ref. 92, and the used water model is SPC/E. The LJ and Coulomb contributions to the free energy are calculated separately so that $F=F_{\mathrm{LJ}}+F_{\mathrm{Coul}}$. The explicit procedure and simulation parameters are explained in Ref. 63. The Coulomb part of the free energy does not only contain the linear polarization contribution as described by the Born solvation model given in Eq. (5), which is proportional to the square of the ion charge, but also a term linear in the ion charge, which corresponds to an electrostatic potential contribution, and non-linear dielectric terms. All these terms can be described by the non-linear dielectric model according to

$$
\begin{aligned}
F_{\text {Coul }} & =\Phi q+A q^{2}+B q^{3}+C q^{4} \\
& =\Phi q+F_{\text {Coul }}^{\text {lin }}+B q^{3}+C q^{4} .
\end{aligned}
$$

Herein, $A$ denotes the coefficient of the linear dielectric contribution, $\Phi$ is the electrostatic potential inside the ionic cavity due to water orientation effects, and $B$ and $C$ are non-linear coefficients. To obtain the four coefficients, we calculate the free energy for different fractions of the full ion charge and fit the resulting Coulomb free energy to Eq. (A1). We first fit up to second order using data within a range of $\pm 0.2 e$ and afterward fit the remaining coefficients with all the data. The fits are shown in Fig. 9, and Table IV shows the fitted coefficients, which for $\mathrm{Cl}^{-}$agree with our previous work. ${ }^{63}$ The errors are estimated by varying the range from $\pm 0.1 e$ to $\pm 0.3 e$ and taking the standard deviation. From the results of the fit, we can estimate the ionic Born diameters $a_{B}$ by using the Born equation, as given in Eq. (5), together with the SPC/E dielectric constant $\varepsilon_{\mathrm{SPC} / \mathrm{E}}$ $=71$. Table $\mathrm{V}$ shows the solvation free energies $F$ and the
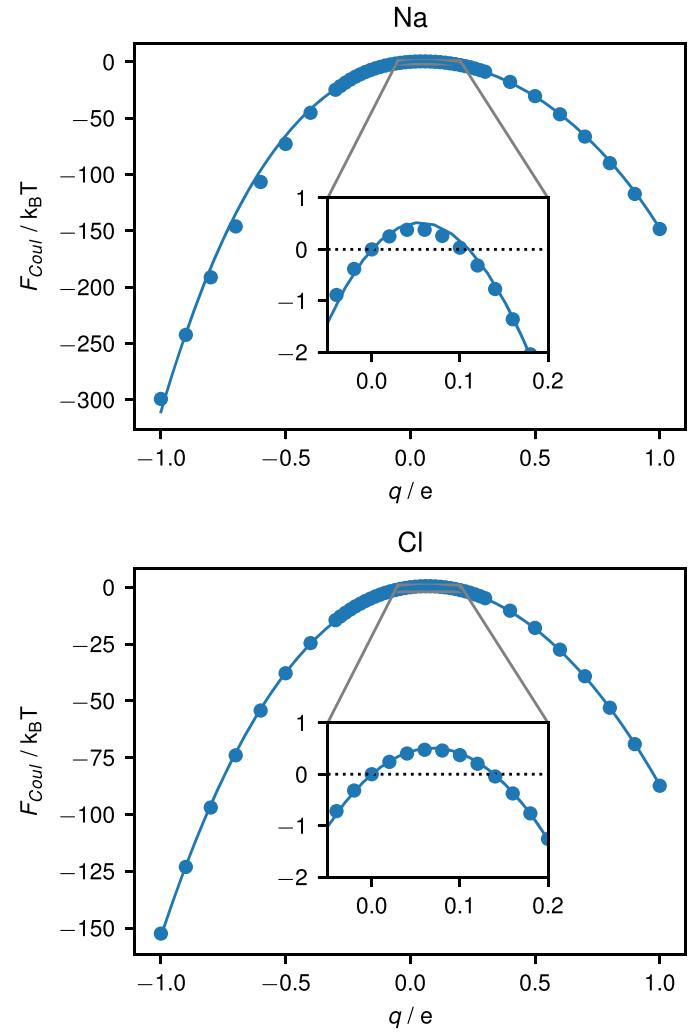

FIG. 9. Coulomb contribution to the free energy for different fractions of a full charged ion. The continuous line shows the fit according to Eq. (A1).

corresponding resultant values of $a_{B}$ separately for the $\mathrm{Na}^{+}$and $\mathrm{Cl}^{-}$ ions. The two diameters are quite similar to each other and agree reasonably well with the fit to experimental data in Table II. However, the Born model only describes the linear part of the Coulomb part of the solvation free energy. When we fit only the Coulombic part $F_{\text {Coul }}$ by the Born model, we obtain the modified ion diameters $a_{\text {Coul }}$, which differ only slightly from the ionic Born diameters $a_{B}$. However, when we fit only the linear Coulombic part $F_{\text {Coul }}^{\text {lin }}=A q^{2}$ by

TABLE IV. Coefficients according to Eq. (A1) in units of $k_{B} T$.

\begin{tabular}{lcccr}
\hline \hline Ion & $\Phi e$ & $A e^{2}$ & $B e^{3}$ & \multicolumn{1}{c}{$C e^{4}$} \\
\hline $\mathrm{Na}^{+}$ & $19 \pm 2$ & $-181 \pm 6$ & $62 \pm 3$ & $-48 \pm 7$ \\
$\mathrm{Cl}^{-}$ & $14.8 \pm 0.4$ & $-109.0 \pm 0.8$ & $18.6 \pm 0.5$ & $-11.5 \pm 1$ \\
\hline \hline
\end{tabular}

TABLE V. Solvation free energies $\left(k_{B} T\right)$ and corresponding ionic diameters $(n m)$ according to Eq. (5).

\begin{tabular}{lcccccc}
\hline \hline Ion & $F$ & $a_{B}$ & $F_{\text {Coul }}$ & $a_{\text {Coul }}$ & $F_{\text {Coul }}^{\text {lin }}$ & $a_{\text {Coul }}^{\text {lin }}$ \\
\hline $\mathrm{Na}^{+}$ & -146.0 & 0.376 & -148.8 & 0.369 & -181 & 0.304 \\
$\mathrm{Cl}^{-}$ & -144.2 & 0.381 & -152.8 & 0.360 & -109.0 & 0.504 \\
\hline \hline
\end{tabular}




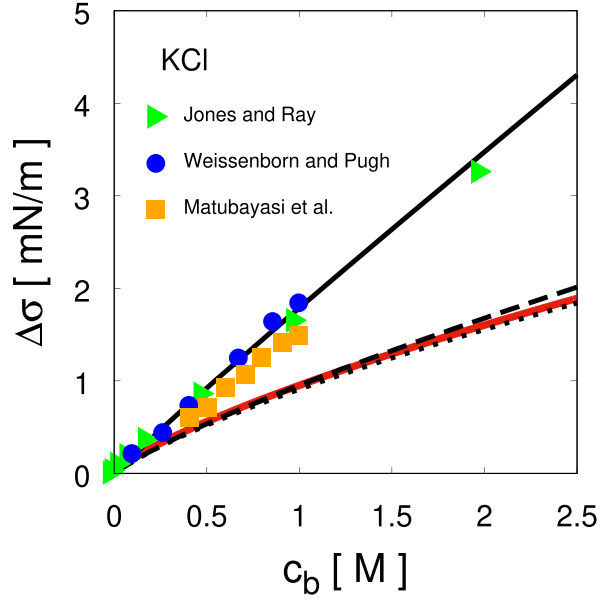

Onsager-Samaras theory

MPB-2, $d=0.230 \mathrm{~nm}, \alpha_{\mathrm{K}}=\alpha_{\mathrm{Cl}}=0.8$

MPB-2, $d=0.230 \mathrm{~nm}, \alpha_{\mathrm{K}}=\alpha_{\mathrm{Cl}}=0.0--$

MPB-2, $d=0 \mathrm{~nm}, \alpha_{\mathrm{K}}=\alpha_{\mathrm{Cl}}=0.0 \cdots$

FIG. 10. Comparison of the MPB-2 model results with the Onsager-Samaras theory. The black solid, broken, and dotted lines follow from the MPB-2 model for different values of $d, \alpha_{\mathrm{K}}$, and $\alpha_{\mathrm{Cl}}$ as described in the legend. The red solid line corresponds to the Onsager-Samaras theory calculated by evaluating the infinite series in Eq. (18) up to $n=100$, which perfectly agrees with the MPB-2 model results for $d=0 \mathrm{~nm}$ and $\alpha_{\mathrm{K}}=\alpha_{\mathrm{Cl}}=0$ (black dotted line). The experimental data deviate significantly from the Onsager-Samaras theory and can be described by the MPB-2 model results when using repulsive ion-interface interactions with a strength of $\alpha_{\mathrm{K}}=\alpha_{\mathrm{Cl}}=0.8$ (solid line).

the Born model, we obtain the modified ion diameters $a_{\text {Coul }}^{\text {lin }}$, which differ significantly from the ionic Born diameters $a_{B}$. This demonstrates that the ionic Born diameters $a_{B}$, which result from fitting the total solvation free energy to the Born equation, as given in Eq. (5), are not physically meaningful since the Born solvation model is not strictly valid even for monovalent ions. The large difference between the ionic Born diameters $a_{B}$ and the interaction ionic diameters $d$ as extracted from a fit of the experimental activity coefficients is therefore not surprising.

\section{APPENDIX B: COMPARISON OF MODIFIED PB MODEL WITH OS THEORY}

Figure 10 shows a comparison of the MPB-2 model results with the Onsager-Samaras theory. The red line is the Onsager-Samaras theory [Eq. (18)], which is equal to the MPB-2 model with $d=0 \mathrm{~nm}$ and $\alpha_{\mathrm{K}}=\alpha_{\mathrm{Cl}}=0$ (the dotted line). With $d=0.230 \mathrm{~nm}$ (the broken line), the MPB-2 model shows a small difference from the OnsagerSamaras theory, but still the experimental data significantly deviate from the broken line. The black solid line is the fit of the MPB-2 model with the experimental data where $d=0.230 \mathrm{~nm}$ and $\alpha_{\mathrm{K}}=\alpha_{\mathrm{Cl}}$ $=0.8$. Here, good agreement with the experimental data is observed.

\section{APPENDIX C: VALIDITY OF MSA THEORY}

In Fig. 11, we show the comparison between MSA theory and GCMC simulations for dielectric decrements corresponding to $\mathrm{NaCl}$ and $\mathrm{NaI}$ for various effective ionic diameters $d$. The agreement between theory and simulations for $\mathrm{NaI}$ using the diameter $d=0.27 \mathrm{~nm}$ (which is the best-fit result from Fig. 3) is not perfect for concentrations above $2 \mathrm{M}$. The validity of MSA theory is governed by the electrostatic coupling parameter, which is the Coulomb energy at contact, given by $e^{2} /\left(4 \pi \varepsilon\left(c_{\mathrm{b}}\right) \varepsilon_{0} k_{\mathrm{B}} T d\right)$. For a concentration of $2 \mathrm{M}$, the dielectric constant of $\mathrm{NaI}$ is rather small and given by $\varepsilon(2 M)=52.7$, which results in a coupling parameter of 3.93 . We thus conclude that for coupling parameters below four, MSA theory reproduces well the GCMC simulation results. For $\mathrm{NaCl}$ and the best-fit diameter $d=0.249 \mathrm{~nm}$, the deviations between MSA and GCMC are less because the dielectric constant is higher, and thus, the electrostatic coupling parameter is smaller.

\section{APPENDIX D: PREDICTIONS FOR ACTIVITY COEFFICIENTS NEGLECTING THE IONIC SOLVATION CONTRIBUTION}

An interesting and somewhat puzzling observation is that the primitive model reproduces the experimental activity coefficient data very well if one neglects the ionic solvation contribution $\beta \mu_{i w}$
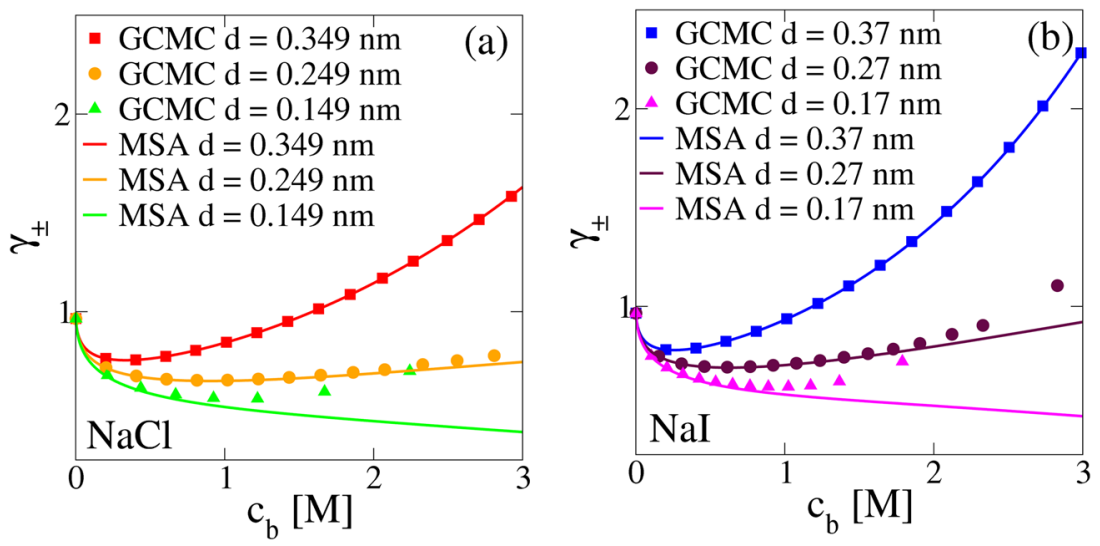

FIG. 11. Mean ionic activity coefficient as a function of salt concentration for (a) $\mathrm{NaCl}$ and (b) Nal. The lines represent MSA theory combined with the Born solvation model [Eq. (2)] including the salt-concentration-dependent dielectric function. Symbols represent GCMC simulation results using the same parameters. 

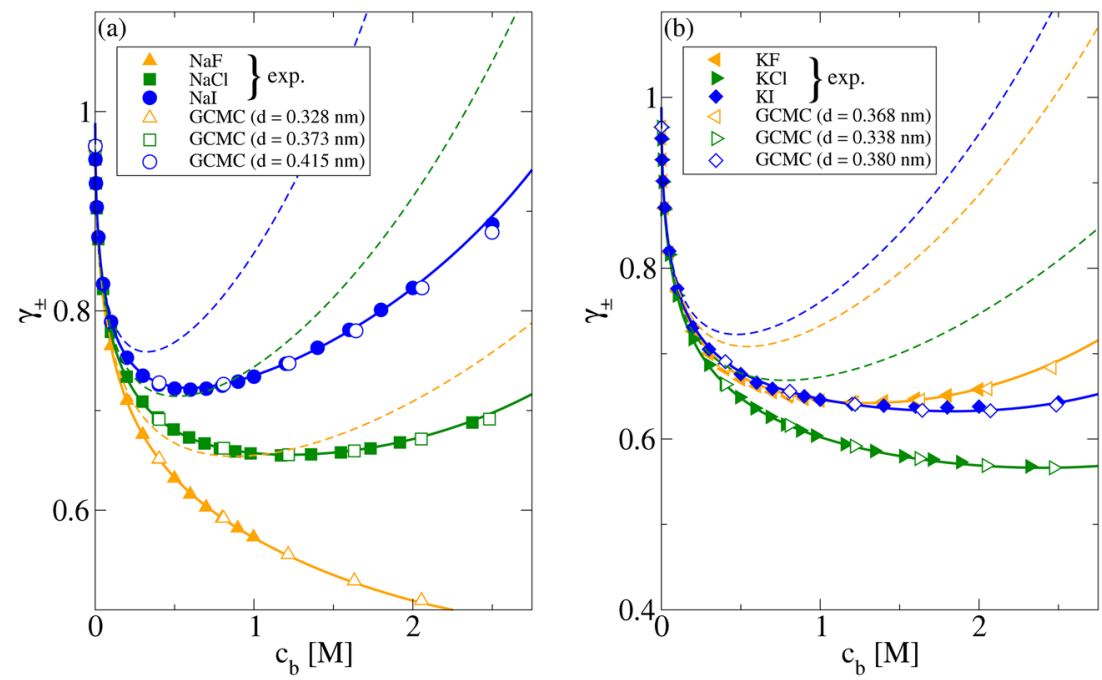

FIG. 12. Mean ionic activity coefficient as a function of salt concentration for (a) $\mathrm{NaF}, \mathrm{NaCl}$, and $\mathrm{Nal}$, and (b) $\mathrm{KF}, \mathrm{KCl}$, and $\mathrm{Kl}$. The experimental data are represented by full symbols. ${ }^{83}$ Here, the theoretical predictions neglect the ionic solvation contribution $\beta \mu_{i w}$ in Eq. (4) to the mean ionic activity coefficient in the MSA result [Eq. (2)] and also in the GCMC simulations. Empty symbols represent the GCMC simulations including fitted ionic diameters $d$ and dielectric molten-salt parameters $\varepsilon_{\mathrm{ms}}$. The solid lines represent MSA theory [Eq. (2)] including the salt-concentration-dependent dielectric function, while the dashed lines represent MSA theory for the same ion diameters $d$ but for a fixed dielectric constant corresponding to pure water. The units were transformed from molality to molarity using experimental solution mass density data for $\mathrm{KCl}^{84}$ and $\mathrm{NaCl}^{85} \mathrm{As}^{8}$ the correction is negligible for $\mathrm{KCl}$ and $\mathrm{NaCl}$, we assume the same to be true also for the other salts (for which no data are available).

in Eq. (4) to the mean ionic activity coefficient in Eq. (2). In Fig. 12, we compare the experimental data with MSA theory predictions as well as GCMC simulations, where as the only fitting parameter we determine the effective mean ionic diameter $d$. Figure 12 is analogous to Fig. 3 where we do include the ionic solvation contribution $\beta \mu_{i w}$ in both the MSA theory and the GCMC simulations. The fitted ionic diameters $d$ in Fig. 12 are different and significantly larger than in Fig. 3. Curiously, the agreement between the experimental data and the MSA and GCMC predictions is much better. In addition, the agreement between the MSA and GCMC predictions is better, which is due to the fact that the fitted diameter values in Fig. 12 are higher.

\section{DATA AVAILABILITY}

The data that support the findings of this study are available within the article.

\section{REFERENCES}

${ }^{1}$ A. Heydweiller, Ann. Phys. 338, 145 (1910).

${ }^{2}$ G. Jones and W. A. Ray, J. Am. Chem. Soc. 59, 187 (1937).

${ }^{3}$ N. L. Jarvis and M. A. Scheiman, J. Phys. Chem. 72, 74 (1968).

${ }^{4}$ P. K. Weissenborn and R. J. Pugh, J. Colloid Interface Sci. 184, 550 (1996).

${ }^{5}$ N. Matubayasi, K. Tsunetomo, I. Sato, R. Akizuki, T. Morishita, A. Matuzawa, and Y. Natsukari, J. Colloid Interface Sci. 243, 444 (2001).

${ }^{6}$ N. Matubayasi, K. Yamamoto, S.-i. Yamaguchi, H. Matsuo, and N. Ikeda, J. Colloid Interface Sci. 214, 101-105 (1999).

${ }^{7}$ W. Kunz, L. Belloni, O. Bernard, and B. W. Ninham, J. Phys. Chem. B 108, 2398 (2004).
${ }^{8}$ T. López-León, A. B. Jódar-Reyes, D. Bastos-González, and J. L. Ortega-Vinuesa, J. Phys. Chem. B 107, 5696 (2003).

${ }^{9}$ T. López-León, M. J. Santander-Ortega, J. L. Ortega-Vinuesa, and D. BastosGonzález, J. Phys. Chem. C 112, 16060 (2008).

${ }^{10}$ J. M. Peula-García, J. L. Ortega-Vinuesa, and D. Bastos-González, J. Phys. Chem. C 114, 11133 (2010).

${ }^{11}$ A. P. dos Santos and Y. Levin, Phys. Rev. Lett. 106, 167801 (2011).

${ }^{12}$ A. P. dos Santos, W. Figueiredo, and Y. Levin, Langmuir 30, 4593 (2014).

${ }^{13}$ N. Jiang, P. Li, Y. Wang, J. Wang, H. Yan, and R. K. Thomas, J. Phys. Chem. B 108, 15385 (2004).

${ }^{14}$ L. S. Romsted, Langmuir 23, 414 (2007).

${ }^{15}$ J. Luczak, M. Markiewicz, J. Thöming, J. Hupka, and C. Jungnickel, J. Colloid Interface Sci. 362, 415 (2011).

${ }^{16}$ W. Müller, C. Déjugnat, T. Zemb, J.-F. Dufrêche, and O. Diat, J. Phys. Chem. B 117, 1345 (2013).

${ }^{17}$ J. Morag, M. Dishon, and U. Sivan, Langmuir 29, 6317 (2013).

${ }^{18}$ T. Oncsik, G. Trefalt, M. Borkovec, and I. Szilagyi, Langmuir 31, 3799 (2015).

${ }^{19}$ Y. von Hansen, I. Kalcher, and J. Dzubiella, J. Phys. Chem. B 114, 13815 (2010).

${ }^{20}$ H. I. Okur, J. Hladílková, K. B. Rembert, Y. Cho, J. Heyda, J. Dzubiella, P. S. Cremer, and P. Jungwirth, J. Phys. Chem. B 121, 1997 (2017).

${ }^{21}$ J. Lyklema, Chem. Phys. Lett. 467, 217 (2009).

${ }^{22}$ W. Kunz, Curr. Opin. Colloid Interface Sci. 15, 34 (2010).

${ }^{23}$ Y. Zhang and P. S. Cremer, Annu. Rev. Phys. Chem. 61, 63 (2010).

${ }^{24}$ G. Trefalt, T. Palberg, and M. Borkovec, Curr. Opin. Colloid Interface Sci. 27, 9 (2017).

${ }^{25}$ M. Boström, D. R. M. Williams, and B. W. Ninham, Langmuir 17, 4475 (2001).

${ }^{26}$ L. Perera and M. L. Berkowitz, J. Chem. Phys. 95, 1954 (1991).

${ }^{27}$ L. X. Dang and D. E. Smith, J. Chem. Phys. 99, 6950 (1993).

${ }^{28}$ P. Jungwirth and D. J. Tobias, J. Phys. Chem. B 105, 10468 (2001).

${ }^{29}$ P. Jungwirth and D. J. Tobias, J. Phys. Chem. B 106, 6361 (2002).

${ }^{30}$ P. Jungwirth and D. J. Tobias, Chem. Rev. 106, 1259 (2006).

${ }^{31}$ D. Horinek and R. R. Netz, Phys. Rev. Lett. 99, 226104 (2007).

${ }^{32}$ Y. Levin, Phys. Rev. Lett. 102, 147803 (2009). 
${ }^{33}$ Y. Levin, A. P. dos Santos, and A. Diehl, Phys. Rev. Lett. 103, 257802 (2009).

${ }^{34}$ D. J. Tobias, A. C. Stern, M. D. Baer, Y. Levin, and C. J. Mundy, Annu. Rev. Phys. Chem. 64, 339 (2013).

${ }^{35}$ Y. Levin and A. P. dos Santos, J. Phys.: Condens. Matter 26, 203101 (2014).

${ }^{36}$ D. Horinek, A. Herz, L. Vrbka, F. Sedlmeier, S. I. Mamatkulov, and R. R. Netz, Chem. Phys. Lett. 479, 173 (2009).

${ }^{37}$ N. Schwierz, D. Horinek, and R. R. Netz, Langmuir 26, 7370 (2010).

${ }^{38}$ S. I. Mamatkulov, C. Allolio, R. R. Netz, and D. J. Bonthuis, Angew. Chem., Int. Ed. 56, 15846 (2017).

${ }^{39}$ K. S. Pitzer, J. Phys. Chem. 77, 268 (1973).

${ }^{40}$ K. S. Pitzer and G. Mayorga, J. Phys. Chem. 77, 2300-2308 (1973).

${ }^{41}$ E. Moggia and B. Bianco, J. Phys. Chem. B 111, 3183 (2007).

${ }^{42}$ C. Held, L. F. Cameretti, and G. Sadowski, Fluid Phase Equilib. 270, 87 (2008).

${ }^{43}$ D. Fraenkel, J. Chem. Theory Comput. 11, 178 (2015).

${ }^{44}$ L. Blum, Mol. Phys. 30, 1529 (1975).

${ }^{45}$ H. R. Corti, J. Phys. Chem. 91, 686 (1987).

${ }^{46}$ T. S. Sorensen, J. B. Jensen, and P. Sloth, J. Chem. Soc., Faraday Trans. 1 85, 2649 (1989).

${ }^{47}$ J. B. Hasted, D. M. Ritson, and C. H. Collie, J. Chem. Phys. 16, 1 (1948).

${ }^{48}$ W. R. Fawcett and A. C. Tikanen, J. Phys. Chem. 100, 4251 (1996).

${ }^{49}$ J.-P. Simonin, L. Blum, and P. Turq, J. Phys. Chem. 100, 7704 (1996).

${ }^{50}$ J.-P. Simonin, J. Phys. Chem. B 101, 4313 (1997).

${ }^{51}$ I. Kalcher and J. Dzubiella, J. Chem. Phys. 130, 134507 (2009).

${ }^{52}$ J. Vincze, M. Valiskó, and D. Boda, J. Chem. Phys. 133, 154507 (2010).

${ }^{53}$ M. Valiskó and D. Boda, J. Chem. Phys. 140, 234508 (2014).

${ }^{54}$ A. Levy, M. Bazant, and A. Kornyshev, Chem. Phys. Lett. 738, 136915 (2020).

${ }^{55}$ A. Levy and D. Andelman, Phys. Rev. Lett. 108, 227801 (2012).

${ }^{56}$ N. Gavish and K. Promislow, Phys. Rev. E 94, 012611 (2016).

${ }^{57}$ C.-H. Ho, H.-K. Tsao, and Y.-J. Sheng, J. Chem. Phys. 119, 2369 (2003).

${ }^{58}$ D. Henderson, D. Gillespie, T. Nagy, and D. Boda, Mol. Phys. 103, 2851 (2005).

${ }^{59}$ A. Diehl, A. P. dos Santos, and Y. Levin, J. Phys.: Condens. Matter 24, 284115 (2012).

${ }^{60}$ L. Onsager and N. N. T. Samaras, J. Chem. Phys. 2, 528 (1934).

${ }^{61}$ R. H. Garrett and C. M. Grisham, Biochemistry (Brooks/Cole, Boston, 2010).

${ }^{62}$ Y. Marcus, J. Chem. Soc., Faraday Trans. 87, 2995 (1991).

${ }^{63}$ P. Loche, C. Ayaz, A. Schlaich, D. J. Bonthuis, and R. R. Netz, J. Phys. Chem. Lett. 9, 6463 (2018).

${ }^{64}$ A. Bakhshandeh, A. P. dos Santos, A. Diehl, and Y. Levin, J. Chem. Phys. 142, 194707 (2015).
${ }^{65}$ M. P. Allen and D. J. Tildesley, Computer Simulations of Liquids (Oxford University Press, Oxford, 1987).

${ }^{66}$ Y. Uematsu, D. J. Bonthuis, and R. R. Netz, J. Phys. Chem. Lett. 9, 189 (2018).

${ }^{67}$ D. Boda, D. Gillespie, W. Nonner, D. Henderson, and B. Eisenberg, Phys. Rev. E 69, 046702 (2004).

${ }^{68}$ S. Tyagi, M. Süzen, M. Sega, M. Barbosa, S. S. Kantorovich, and C. Holm, J. Chem. Phys. 132, 154112 (2010).

${ }^{69}$ V. Jadhao, F. Solis, and M. de la Cruz, Phys. Rev. Lett. 109, 223905 (2012).

${ }^{70} \mathrm{~J}$. W. Zwanikken and M. Olvera de la Cruz, Proc. Natl. Acad. Sci. U. S. A. 110, 5301 (2013).

${ }^{71}$ Z. Gan, H. Wu, K. Barros, Z. Xu, and E. Luijten, J. Comput. Phys. 291, 317 (2015).

${ }^{72}$ A. P. dos Santos, M. Girotto, and Y. Levin, J. Chem. Phys. 147, 184105 (2017).

${ }^{73}$ A. P. dos Santos and Y. Levin, J. Chem. Phys. 142, 194104 (2015).

${ }^{74}$ A. P. dos Santos, M. Girotto, and Y. Levin, J. Phys. Chem. B 120, 10387 (2016).

${ }^{75}$ P. W. Debye and E. Hückel, Phys. Z 24, 185 (1923).

${ }^{76}$ Y. Levin, Rep. Prog. Phys. 65, 1577 (2002).

${ }^{77}$ R. Buchner, G. T. Hefter, and J. Barthel, J. Chem. Soc., Faraday Trans. 90, 2475 (1994).

${ }^{78}$ R. Buchner, G. T. Hefter, and P. M. Ma, J. Phys. Chem. A 103, 1 (1999).

${ }^{79}$ J. Barthel, J. Krüger, and E. Schollmeyer, Z. Phys. Chem. 104, 59 (1977).

${ }^{80}$ A. V. Kobelev, A. S. Lileev, and A. K. Lyashchenko, Russ. J. Inorg. Chem. 56, 652 (2011).

${ }^{81}$ E. Glueckauf, Trans. Faraday Soc. 60, 1637 (1964).

${ }^{82}$ A. Chandra, J. Chem. Phys. 113, 903 (2000).

${ }^{83}$ W. J. Hamer and Y. C. Wu, J. Phys. Chem. Ref. Data 1, 1047 (1972).

${ }^{84}$ M. T. Zafarani-Moattar and F. Izadi, J. Chem. Thermodyn. 43, 552 (2011).

${ }^{85}$ P. S. Z. Rogers and K. S. Pitzer, J. Phys. Chem. Ref. Data 11, 15 (1982).

${ }^{86}$ V. Balos, M. Bonn, and J. Hunger, Phys. Chem. Chem. Phys. 19, 9724 (2017).

${ }^{87}$ N. Schwierz, D. Horinek, U. Sivan, and R. R. Netz, Curr. Opin. Colloid Interface Sci. 23, 10 (2016).

${ }^{88}$ J. M. Barthel, H. Krienke, and W. Kunz, Physical Chemistry of Electrolyte Solutions: Modern Aspects (Springer, New York, 1998).

${ }^{89}$ K. Ali, A. A. Shah, S. Bilal, and A. A. Shah, Colloids Surf., A 337, 194 (2009).

${ }^{90}$ L. Piatkowski, Z. Zhang, E. H. Backus, H. J. Bakker, and M. Bonn, Nat. Commun. 5, 4083 (2014).

${ }^{91}$ T. Markovich, D. Andelman, and R. Podgornik, J. Chem. Phys. 142, 044702 (2015).

${ }^{92}$ D. E. Smith and L. X. Dang, J. Chem. Phys. 100, 3757 (1994). 